\title{
Regional variations in the chemical and helium-carbon isotope composition of geothermal fluids across Tunisia
}

\author{
E. Fourré ${ }^{\text {a,* }}$, R. Di Napoli ${ }^{\text {b }}$, A. Aiuppa ${ }^{\text {b,c }}$, F. Parello ${ }^{\text {b }}$, E. Gaubi ${ }^{\text {d }}$, P. Jean-Baptiste ${ }^{\text {a }}$, P. Allard ${ }^{\text {e }}$, \\ S. Calabrese ${ }^{\mathrm{b}}, \mathrm{A}$. Ben Mamou ${ }^{\mathrm{d}}$ \\ a LSCE, CEA-Saclay, Gif-sur-Yvette, France \\ b DiSTeM, Università di Palermo, Italy \\ c Istituto Nazionale di Geofisica e Vulcanologia, Sezione di Palermo, Italy, Italy \\ d Laboratoire Ressources Minérales et Environnement, Univ. Tunis El-Manar, Tunisia \\ e IPGP, Paris, France
}

\section{A R T I C L E I N F O}

\section{Article history:}

Received 17 January 2011

Received in revised form 19 May 2011

Accepted 7 July 2011

Available online 19 July 2011

\section{Keywords:}

Tunisia

Helium isotopes

Carbon isotopes

Geothermal fluids

Groundwaters

Thermal springs

\begin{abstract}
A B S T R A C T
Tunisia has numerous thermo-mineral springs. Previous studies have shown that their chemical composition and occurrence are strongly influenced by the regional geology. However little work has been done so far to study the isotopic composition of volatiles associated with these geothermal manifestations. Here, we report on the results of an extensive survey of both natural hot springs and production wells across Tunisia, aimed at investigating the spatial distribution of thermal fluids' geochemical characteristics and $\mathrm{He}-\mathrm{C}$ isotopic composition. The chemistry of the analyzed samples highlights the heterogeneity of the water mineralization processes in Tunisia, as a consequence of the complex geological and tectonic setting of the country. In terms of chemical composition, we are able to conclude, however, that dissolution of halite and gypsum plays a key control on groundwater chemistry. Helium and carbon isotope systematics confirm the prevalently crustal origin of the volatiles interacting with the aquifer systems, consistent with the absence of any recent magmatism. Most samples are characterized by crustal-type helium $\left({ }^{3} \mathrm{He} /{ }^{4} \mathrm{He}\right.$ in the range $\left.0.02 \mathrm{Ra}-0.4 \mathrm{Ra}\right)$ associated with $\mathrm{CO}_{2}$ predominantly metamorphic in origin (with the exception of the Saharan platform where the carbon content is low and mostly organically-derived). In Eastern Tunisia, however, a few samples have $\mathrm{He}-\mathrm{C}$ isotope compositions which suggest (at least partial) mantle derivation of the dissolved gas phase : the ${ }^{3} \mathrm{He} /{ }^{4} \mathrm{He}$ ratio reaches $2.4 \mathrm{Ra}$ (corresponding to $30 \%$ of mantle-derived helium) at the Ain Garci site, a $\mathrm{CO}_{2}$ rich mineral spring located some $30 \mathrm{~km}$ south of the city of Zaghouan. This mantle signature is consistent with the fact that the Pelagian Block, to which Eastern Tunisia belongs, has been deeply affected by extensional and transtensional tectonics since the opening of the Tethys, a process which is still ongoing in the Sicily channel (Pantelleria Rift). As a whole however, our results show that the Italian mantle gas anomaly only marginally extends to Northwestern Africa.
\end{abstract}

(c) 2011 Elsevier B.V. All rights reserved.

\section{Introduction}

Tectonically- and seismically-active regions of the world have long been known for being sites of widespread release of deep-rising fluids and associated volatiles (Irwin and Barnes, 1980). In the central Mediterranean for instance, studies of the Italian peninsula have shown that, in addition to the huge gas emissions from active volcanism (Allard et al., 1991; Aiuppa et al., 2008), large amounts of volatiles (e.g., $>2 \cdot 10^{11} \mathrm{~mol} /$ year of $\mathrm{CO}_{2}$; Chiodini et al., 2004, 2008) are also released in diffuse form through the tectonically active Tyrrhenian segment of the country. Such volatile degassing occurs in either diffuse form (through regional aquifers), or diffuse soil

\footnotetext{
* Corresponding author.

E-mail address: Elise.Fourre@lsce.ipsl.fr (E. Fourré).
}

degassing, or in localized (spot) manifestations, either cold or hot (fumaroles, hot springs, etc.). The question therefore arises whether, and to what extent, such degassing pattern also extends to nearby north-western Africa, an area which has undergone similarly complex tectonic evolution since the Mesozoic.

Tunisia, in particular, has numerous thermo-mineral springs, the majority of which are used for public baths (hammam), swimming pools, and for medical treatments provided by thermal establishments. Previous studies (Meddeb, 1993; Sadki, 1998) have shown that location and chemical composition of these thermal manifestations are strongly influenced by the regional geology. However, while geology and tectonism in Tunisia have long been a subject of interest, little work has been conducted so far to investigate the isotopic composition of volatiles associated with these geothermal manifestations, and their relation with the geological and structural framework of Tunisia. 
Many studies have demonstrated that the characterization of the $\mathrm{He}-\mathrm{CO}_{2}$ relative abundances and isotopic compositions in geothermal manifestations can valuably assist in constraining the origin of the fluids (Marty and Jambon, 1987; Griesshaber et al., 1992; Lollar et al., 1997; Hulston et al., 2001; de Leeuw et al., 2007; Mutlu et al., 2008; Lollar and Ballentine, 2009; Mata et al., 2010). In this respect, because they vary by more than three orders of magnitude in terrestrial samples, helium isotopes are particularly good indicators of both the extent of mantle-derived contributions to crustal fluids and the rate of mantle degassing through the crust (Ballentine et al., 1991; Marty et al., 1992; Ballentine et al., 2002; Güleç et al., 2002; Kipfer et al., 2002 and references therein). This results from the distinct origins of ${ }^{3} \mathrm{He}$ (essentially primordial) and ${ }^{4} \mathrm{He}$ (produced by the radioactive decay of $U$ and $T h$ ) and their contrasted proportions in Earth's reservoirs. When referred to the atmospheric ratio $\left(R_{a}=1.38 \times 10^{-6}\right)$, typical ${ }^{3} \mathrm{He} /{ }^{4} \mathrm{He}$ ratios vary from $<0.05 \mathrm{R}_{\mathrm{a}}$ in the continental crust to $8 \pm 1 \mathrm{R}_{\mathrm{a}}$ on average in the upper mantle, and up to $40-50 R_{a}$ in products of plume-related ocean islands, such as Hawaii and Iceland (Marty et al., 1991, 1996; Ballentine and Burnard, 2002; Graham, 2002; Hilton et al., 2002; Stuart et al., 2003). Spatial variations of the ${ }^{3} \mathrm{He} /{ }^{4} \mathrm{He}$ ratios can thus be used to identify contemporary magmatic activity and/or tectonic processes that may facilitate the transport of mantle volatiles to shallow crustal levels (Polyak et al., 2000; Ballentine et al., 2002; Caracausi et al., 2005; Pik and Marty, 2009) and provide valuable information about regional tectonic environments. The application of the above "isotopic approach" to Italian gas discharges for instance, has revealed a complex scenario in which an heterogeneous (variably metasomatized) mantle, and gas production in the crust, both contribute to the source of the surface gas anomaly (e.g., Chiodini et al., 2000, 2004; Parello et al., 2000; Martelli et al., 2004, 2008).

Here, we report on the results of a survey, carried out in 2006 and 2009 , of both production wells and natural hot springs across Tunisia. A total of 43 springs and wells were sampled for carbon and helium isotope geochemistry to map the spatial distribution of $\delta^{13} \mathrm{C}_{\text {TDIC }}$ (TDIC stands for Total Dissolved Inorganic Carbon) and ${ }^{3} \mathrm{He} /{ }^{4} \mathrm{He}$ ratios. In addition to water samples, gas samples were also collected and analyzed for helium isotopes whenever gas bubbles were present. At each site, samples were also taken for chemical analysis and stable isotope geochemistry $(\mathrm{O}, \mathrm{H})$. The aim of this work is to determine helium and associated carbon isotopic composition of the main geothermal areas of Tunisia in order to gain insights into the subsurface physical and chemical processes determining the geochemistry of Tunisian hydrothermal manifestations and their links with the complex neotectonic framework of the country.

\section{The study area}

\subsection{Geological and tectonic settings}

Tunisia belongs to the North African margin (Fig. 1). It occupies a peculiar position at the boundary between the stable Precambrian Saharan domain and the younger and deformed Atlasic domain. A complex tectonic evolution, resulting from interactions between the African and European plates, has affected Tunisia since the Late Permian, at the beginning of Pangea breakup, and until the Cenozoic Alpine orogeny of the Maghrebide chain (Bouaziz et al., 2002). As a result, the geology of Tunisia is of remarkable complexity.

Three main structural domains can be identified in Tunisia : the Saharan, the Atlasic and the Eastern domains (Fig. 1). The Saharan domain, in southern Tunisia, forms a stable tabular zone which consists of a granitic and metamorphic basement (Laaridhi-Ouazaa, 1994), overlain by a thin and relatively undeformed sequence of Paleozoic sandstones and carbonates, and Mesozoic evaporites (Ben Ferjani et al., 1990). In its southernmost part, the Saharan plateau is covered by the dunes of the Grand Erg Oriental, while in the north, the

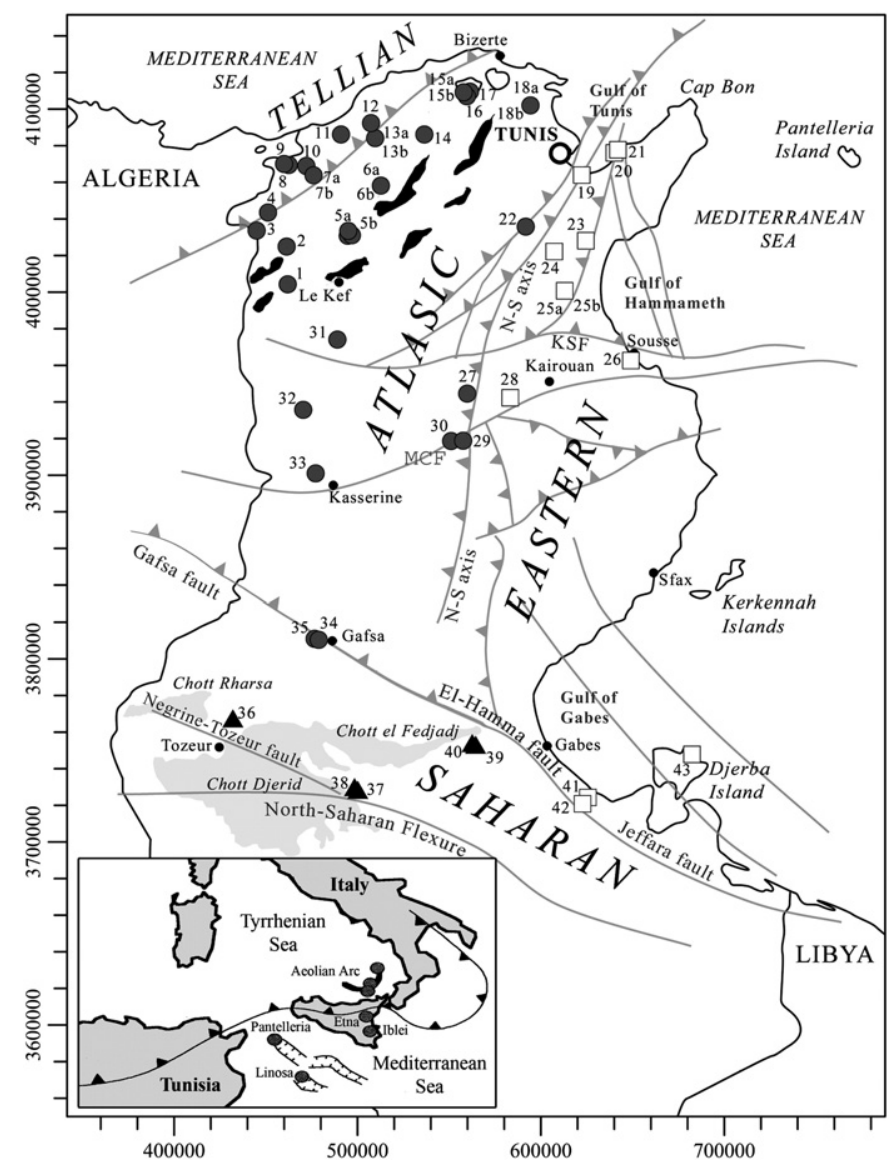

Fig. 1. Simplified structural map of Tunisia, showing its main structural domains (see Section 2): the Saharan domain, the Atlasic domain, and the Eastern domain. The Tellian sector is identified as the NW portion of the Atlasic domain. NE-SW trending Triassic "diapir zone", from Tunis to Le Kef, is marked in black. Sampling sites (numbered as in Table 1) are shown with different symbols for samples collected in the different geographical districts : samples from the Atlasic domain are represented by circles, from the Eastern domain by squares, and from the Saharan domain by triangles. The small inset puts Northern Tunisia in a broader geodynamic context, and shows the collisional boundary between African and European plates, as well as the sites of active volcanism in Sicily and Eolian Islands (black dots).

Modified from Khomsi et al. (2007).

Chotts region comprised between the North Saharan Flexure (Gabtni et al., 2005) and the Gafsa-Jeffara fault system separates this Saharan domain from the thick and folded sedimentary cover of the Atlasic domain (Fig. 1). To the north of the Saharan Platform, the Atlasic domain, with the meridional, central and northern Atlas, occupies most of central Tunisia. It consists of a complex sequence of dominantly SE-verging tectonic depressions and uplifts that developed during the late Cretaceous collision between the African and European plates. It includes the main mountains and ranges of Tunisia, which were formed in a compressive context since the Late Eocene. This domain extends up to the northern coast of Tunisia, while, on its eastern side, it is limited by the North-South Axis (Fig. 1), a major structural lineament trending N-S from the Gulf of Tunis and corresponding to a deep fault system oriented N-S (Burollet, 1956). Thick and well-deformed series of Cretaceous and Tertiary sediments, formed during the Atlas orogeny, make up the Atlasic domain. Marine carbonates with significant fossiliferous content and secondary dolomite, clay and gypsum formations, outcrop in the southernmost part of the Atlasic domain (Gafsa zone, and Kasserine "island"). Further to the north, the Atlasic domain grades to deep marine basin deposits, named "Tunisian Trough", consisting of Cretaceous and Tertiary shales and carbonate formations. In this area, NE-SW 
trending folds and fractures give rise to widespread extrusions of evaporites from the Triassic basement (Perthuisot, 1978; Mlayah et al., 2009); finally giving rise to a $1000 \mathrm{~m}$ thick "diapir zone" (Fig. 1) mainly composed of gypsum and anhydrite, with interbedded clays and thin dolomite layers. This evaporitic sequence, which was deposited during the Late Triassic-Liassic in an epicontinental domain, thickens towards the north, and grades to evaporitic series including abundant halite (Ben Ferjani et al., 1990). To the extreme northwest, the Tellian sector (Fig. 1) occupies the eastern part of the "Maghrebides" fold-thrust belt. It corresponds to a "nappe" domain (Ben Ferjani et al., 1990) made of several geological units displaced southeastward by the tectonic activity (Rouvier, 1977). It was formed during the Late Miocene major orogeny, and its uppermost part is composed of thick (3000-4000 m) series of sandstones and shales belonging to the Oligo-Miocene Numidian Flysch units (Rouvier, 1977). The recent tectonic events in the area are thought to be responsible for its relatively high geothermal gradient $\left(8-9^{\circ} \mathrm{C} / 100 \mathrm{~m}\right)$ and associated high density of thermal manifestations (Meddeb, 1993).

The Eastern domain (Fig. 1) constitutes the young eastern continental margin of Tunisia. It belongs to the Pelagian block, which extends from Tunisia to Sicily, and is characterized by strong extensional tectonics (Pantelleria rift zone) and present-day magmatic activity. Salt pans (sebkhas) and plains (e.g. Kairouan and Sfax plains) characterize this flat area, which was protected from the Atlasic compression by the N-S axis. Since late Cretaceous times, it has followed its own geodynamic evolution and is affected by important tectonic activity of transtensional and transpressional $\mathrm{N}-\mathrm{S}$ and $\mathrm{E}-\mathrm{W}$ deep-seated faults, giving rise to a mosaic of Mesozoic and Cenozoic grabens, horsts, platforms and folds (Bédir, 1995, 1999)

Basic and acidic igneous rocks are widespread all over Tunisia (Ben Ferjani et al., 1990), testifying that volcanic activity has periodically occurred since the Jurassic-Early Cretaceous. The mafic volcanic products are related to the Mio-Pliocene extensional phase, while the more sialic products can be ascribed to shallow crust melting processes during the Miocene compression (Mauduit, 1978).

\subsection{Hydrogeological setting}

The southern Tunisia deep groundwater system is part of the "Continental Intercalaire" aquifer (CI), one of the largest confined aquifers in the world (Castany, 1982), comparable in scale to the Great Artesian basin of Australia. It covers an area of $\sim 600,000 \mathrm{~km}^{2}$ from western Algeria to the Lybian desert and, from north to south, from the Saharan Atlas to the Tassili. It is divided into two sub-basins by the M'zab dorsal : the eastern sub-basin flows towards its natural discharge area of the Tunisian Chotts region and of the Gulf of Gabès, where its mixes with the local Djeffara aquifer. The $\mathrm{CI}$ consists of several water horizons with strong artesian pressure (5-25 bars), high temperatures $\left(65-75^{\circ} \mathrm{C}\right)$ and prevalent $\mathrm{Na}-\mathrm{SO}_{4}-\mathrm{Cl}$ composition (Edmunds et al., 2003). The groundwater reservoir of the $\mathrm{Cl}$ is hosted by the continental formations of the Lower Cretaceous (Neocomian, Barremian, Aptian and Albian). The geology of the CI has been described by Cornet (1964), Bishop (1975) and Castany (1982); the most significant formations include limestones, dolomites, clays, sands, and gypsum-bearing evaporites (Bouri et al., 2008).

Elsewhere in Tunisia, groundwater origin is much more local. Rainwaters infiltrate into the crust, dissolve salts, and warm up under the effect of the geothermal heat flux. Although water emergences are found in all types of sedimentary terrains from the Lias to the Quaternary, most of them coincide with the permeable formations of the upper Eocene and the Oligocene, often in association with tectonic accidents and deep fractures (Jellouli, 2002).

\section{Sampling and methods}

A first sampling campaign for isotopic and geochemical analyses was performed during September 2006. A second campaign took place in October 2009 to complete the initial data set, including seven sites already sampled in 2006. In addition to water samples, gas samples were also collected for helium isotopes whenever gas bubbles were present (five sites). Location of the sampling points is given in Table 1, and displayed on the schematic map of Tunisia (Fig. 1).

Results of analytical determinations are listed in Tables 1 and 2. Discharge temperature, $\mathrm{pH}$, Eh and conductivity were measured in the field using portable instruments. Samples for laboratory chemical analyses were collected and stored in LDPE (low density polyethylene) bottles in order to measure major and minor ion concentrations. Analytical determinations were carried out at Instituto Nazionale di Geofisica e Vulcanologia (INGV), sezione di Palermo. The $\mathrm{HCO}_{3}$ content was determined by volumetric titration with $\mathrm{HCl} 0.1 \mathrm{~N}$ as titrating reagent and methylorange as indicator. Chemical analyses of major constituents were performed by ion-chromatography (Dionex DX120) using specific columns for anions (AS14A) and cations (CS12A). Filtered $(0.45 \mu \mathrm{m})$ and acidified (to $\mathrm{pH}=2$ with ultra-pure $\mathrm{HNO}_{3}$ ) samples were used to determine $\mathrm{Na}, \mathrm{K}, \mathrm{Mg}$ and $\mathrm{Ca}$ concentrations, while anion species $\left(\mathrm{F}, \mathrm{Cl}, \mathrm{Br}, \mathrm{NO}_{3}, \mathrm{SO}_{4}\right)$ were determined on untreated samples. The quality of sample analysis was assessed from ionic balance : all samples exhibited imbalances below $\pm 3 \%$.

The composition of dissolved gasses $\left(\mathrm{O}_{2}, \mathrm{~N}_{2}, \mathrm{CO}_{2}, \mathrm{CH}_{4}, \mathrm{H}_{2}\right.$ and $\left.\mathrm{CO}\right)$ was determined (also at INGV sezione di Palermo) following the procedures described in Capasso and Inguaggiato (1998) and Capasso et al. (2000), using a Perkin Elmer Auto system XLClarus 500 gas chromatographer equipped with Carboxen 1000 columns. A thermal conductivity detector (HWD) was used to measure the concentrations of $\mathrm{H}_{2}, \mathrm{O}_{2}, \mathrm{~N}_{2}$ and $\mathrm{CO}_{2}$, while $\mathrm{CH}_{4}$ and $\mathrm{CO}$ were determined by a flame ionization detector (FID). In Table 2, we report the volumetric percentages (\% vol.) in the dry gas phase of the various gaseous species in equilibrium with each collected water sample, calculated at the sampling temperature according to Capasso and Inguaggiato (1998).

The isotopic composition of Total Dissolved Inorganic Carbon (TDIC) was determined at INGV sezione di Palermo, after purification of the stripped $\mathrm{CO}_{2}$, using a Finningan Delta Plus mass spectrometer. The isotopic value of $\delta^{13} C_{\text {TDIC }}$ (see Table 1), calculated according to the method proposed by Favara et al. (2002), are expressed as $\delta$ \% vs. PDB, with an accuracy of $\pm 0.1 \%$.

Hydrogen and oxygen isotope analyses were performed at LSCE (Saclay) using the uranium reduction and the $\mathrm{CO}_{2}$ equilibration techniques, respectively. The experimental precision of the isotopic analysis is $0.8 \%$ for $\delta \mathrm{D}$ and $0.05 \%$ or for $\delta^{18} \mathrm{O}$ (Stievenard et al., 1994; Bourg et al., 2001).

Water samples for helium isotopes were collected in standard refrigeration grade $3 / 8^{\prime \prime}$ copper tubes equipped with metal clamps at both ends. The tubes were flushed with the water prior to closure using either the natural water flow or a perilstaltic pump connected with Tygon tubing. When present, gas bubbles in water were also collected in copper tubes using an inverted funnel. The helium and neon isotopic analyses were carried out at LSCE (Saclay) with a MAP215 mass spectrometer using standard procedures (Jean-Baptiste et al., 1992). Gas samples in copper tubes were directly connected to the high-vacuum inlet system of the mass spectrometer. Helium and neon dissolved in the water samples were first extracted under vacuum into sealed glass tubes (Jean-Baptiste et al., 1992). Typical ${ }^{4} \mathrm{He}$ and ${ }^{20} \mathrm{Ne}$ blanks are $5 \times 10^{-10}$ and $3 \times 10^{-10} \mathrm{~cm}^{3}$ STP for the mass spectrometer inlet, and $2 \times 10^{-9}$ and $1 \times 10^{-9} \mathrm{~cm}^{3}$ STP for the extraction system, respectively. The accuracy is $\pm 1 \%$ for helium and neon concentrations in both water and gas samples. The analytical 
Table 1

Sample details including sampling location, water chemistry and stable isotopes.

\begin{tabular}{|c|c|c|c|c|c|c|c|c|c|c|c|c|c|c|c|c|c|c|c|c|c|c|}
\hline \multirow[t]{2}{*}{ Sample } & \multirow[t]{2}{*}{ Site name (location $\mathrm{N}, \mathrm{E}$ ) } & \multirow{2}{*}{$\begin{array}{l}\text { Sampling } \\
\text { date }\end{array}$} & \multirow{2}{*}{$\frac{\mathrm{T}}{{ }^{\circ} \mathrm{C}}$} & \multirow[t]{2}{*}{$\mathrm{pH}$} & \multirow{2}{*}{$\frac{\text { cond. }}{\mathrm{mS} / \mathrm{cm}}$} & \multirow{2}{*}{$\frac{\mathrm{Eh}}{\mathrm{mV}}$} & \multirow{2}{*}{$\frac{\mathrm{Na}}{\mathrm{meq} / \mathrm{l}}$} & \multirow{2}{*}{$\frac{\mathrm{K}}{\mathrm{meq} / \mathrm{l}}$} & \multirow{2}{*}{$\frac{\mathrm{Mg}}{\mathrm{meq} / \mathrm{l}}$} & \multirow{2}{*}{$\frac{\mathrm{Ca}}{\mathrm{meq} / \mathrm{l}}$} & \multirow{2}{*}{$\frac{\mathrm{F}}{\mathrm{meq} / \mathrm{l}}$} & \multirow{2}{*}{$\frac{\mathrm{Cl}}{\mathrm{meq} / \mathrm{l}}$} & \multirow{2}{*}{$\frac{\mathrm{Br}}{\mathrm{meq} / \mathrm{l}}$} & \multirow{2}{*}{$\frac{\mathrm{NO}_{3}}{\mathrm{meq} / \mathrm{l}}$} & \multirow{2}{*}{$\frac{\mathrm{SO}_{4}}{\mathrm{meq} / \mathrm{l}}$} & \multirow{2}{*}{$\frac{\text { Alk }}{\mathrm{meq} / \mathrm{l}}$} & \multirow{2}{*}{$\frac{\mathrm{TDS}}{\mathrm{mg} / \mathrm{l}}$} & \multirow{2}{*}{$\frac{\delta^{18} \mathrm{O}}{\%}$} & \multirow{2}{*}{$\frac{\delta \mathrm{D}}{\%}$} & \multirow{2}{*}{$\frac{\text { SI }}{\text { halite }}$} & \multirow{2}{*}{$\frac{\text { SI }}{\text { gypsum }}$} & \multirow{2}{*}{$\frac{\text { SI }}{\text { calcite }}$} \\
\hline & & & & & & & & & & & & & & & & & & & & & & \\
\hline \multicolumn{23}{|c|}{ Atlasic samples } \\
\hline 1 & $\begin{array}{l}\text { H. Mallègue } \\
\quad 4004342,461841\end{array}$ & $13 / 10 / 2006$ & 41.8 & 6.64 & 20.7 & -48 & $1.17 \mathrm{E}+02$ & 0.84 & 6.85 & 14.7 & 0.002 & $1.17 \mathrm{E}+02$ & b.d.l. & b.d.l. & 8.67 & 8.8 & $8.19 \mathrm{E}+03$ & -6.75 & -42.0 & -3.73 & -1.59 & 0.23 \\
\hline 2 & $\begin{array}{l}\text { H. Bezzaz } \\
\quad 4025220,461206\end{array}$ & $12 / 10 / 2006$ & 48.5 & 6.53 & 33.5 & 49.5 & $1.68 \mathrm{E}+02$ & 1.60 & 9.57 & 23.1 & 0.002 & $1.81 \mathrm{E}+02$ & b.d.l. & b.d.l. & 18.1 & 16.2 & $1.28 \mathrm{E}+04$ & -6.94 & -40.1 & -3.43 & -1.22 & 0.58 \\
\hline 3 & $\begin{array}{l}\text { H. Ouchtata } \\
\text { 4033459, } 444894\end{array}$ & 29/09/2009 & 42.9 & 7.04 & 2.33 & -180 & $1.54 \mathrm{E}+01$ & 0.15 & 0.34 & 1.37 & 0.056 & $4.98 \mathrm{E}+00$ & b.d.l. & 0.03 & 0.81 & 11.3 & $1.30 \mathrm{E}+03$ & -7.14 & -36.8 & -5.86 & -2.52 & -0.03 \\
\hline 4 & $\begin{array}{l}\text { H. Ali Daoua } \\
\text { 4043096, } 450931\end{array}$ & $12 / 10 / 2006$ & 39.5 & 6.39 & 99.7 & 93 & $7.07 \mathrm{E}+02$ & 3.52 & 20.0 & 51.0 & 0.002 & $7.32 \mathrm{E}+02$ & b.d.l. & b.d.l. & 58.7 & 10.6 & $4.71 \mathrm{E}+04$ & -6.52 & -33.6 & -2.28 & -0.83 & 0.23 \\
\hline $5 a$ & $\begin{array}{l}\text { H. Biadha } \\
\quad \text { 4031101, } 495048\end{array}$ & $10 / 10 / 2006$ & 40.6 & 6.07 & 94 & -107 & $6.80 \mathrm{E}+02$ & 5.56 & 28.7 & 64.0 & 0.002 & $6.75 E+02$ & b.d.l. & 3.67 & 79.4 & 26.2 & $4.71 \mathrm{E}+04$ & -6.85 & -37.5 & -2.34 & -0.62 & 0.4 \\
\hline $5 b$ & $\begin{array}{l}\text { H. Biadha } \\
\quad 4031101,495048\end{array}$ & 29/09/2009 & 40.6 & 6.06 & 48.5 & -52 & $7.73 \mathrm{E}+02$ & 4.85 & 29.9 & 80.2 & n.d. & $8.00 \mathrm{E}+02$ & b.d.l. & b.d.l. & 72.8 & 26.0 & $5.34 \mathrm{E}+04$ & -6.89 & -34.5 & -2.16 & -0.04 & 0.47 \\
\hline $6 a$ & $\begin{array}{l}\text { H. Salaya } \\
\quad 4058317,512871\end{array}$ & 10/10/2006 & 45.0 & 6.83 & 32.7 & -77.5 & $1.96 \mathrm{E}+02$ & 1.07 & 9.31 & 13.7 & 0.002 & $2.05 \mathrm{E}+02$ & b.d.l. & b.d.l. & 18.5 & 9.9 & $1.37 \mathrm{E}+04$ & -6.09 & -35.7 & -3.34 & -0.93 & 0.24 \\
\hline $6 b$ & $\begin{array}{l}\text { H. Salaya } \\
\quad 4058317,512871\end{array}$ & $28 / 09 / 2009$ & 43.1 & 6.75 & 30.1 & 20 & $1.99 \mathrm{E}+02$ & 0.55 & 7.22 & 14.4 & n.d. & $1.86 \mathrm{E}+02$ & b.d.l. & 1.24 & 17.2 & 9.8 & $1.31 \mathrm{E}+04$ & -6.07 & -36.5 & -3.31 & -1.44 & 0.39 \\
\hline $7 a$ & $\begin{array}{l}\text { H. Es-Salhine } \\
\quad 4063873,476205\end{array}$ & $12 / 10 / 2006$ & 73.4 & 6.76 & 3.53 & -290 & $1.03 \mathrm{E}+01$ & 0.19 & 1.02 & 3.16 & 0.026 & $9.79 \mathrm{E}+00$ & b.d.l. & 0.07 & 0.60 & 3.9 & $9.39 \mathrm{E}+02$ & -6.75 & -34.9 & -5.79 & -2.71 & -0.02 \\
\hline $7 \mathrm{~b}$ & $\begin{array}{l}\text { H. Es-Salhine } \\
\text { 4063873, } 476205\end{array}$ & 29/09/2009 & 72.9 & 6.74 & 3.27 & -50 & $9.89 \mathrm{E}+00$ & 0.14 & 1.00 & 3.11 & 0.012 & $9.63 \mathrm{E}+00$ & b.d.l. & b.d.l. & 0.68 & 3.8 & $9.14 \mathrm{E}+02$ & -7.03 & -41.6 & -5.82 & -2.18 & -0.05 \\
\hline 8 & $\begin{array}{l}\text { H. Bourguiba ("populaire") } \\
\text { 4069236, } 462453\end{array}$ & $12 / 10 / 2006$ & 47.5 & 6.98 & 2.94 & 50 & $1.21 \mathrm{E}+01$ & 0.55 & 1.24 & 3.68 & 0.074 & $7.91 \mathrm{E}+00$ & b.d.l. & b.d.l. & 0.08 & 9.3 & $1.24 \mathrm{E}+03$ & -6.73 & -34.0 & -5.74 & -3.63 & 0.38 \\
\hline 9 & $\begin{array}{l}\text { H. Bourguiba ("basse") } \\
\text { 4069316, } 462469\end{array}$ & $12 / 10 / 2006$ & 34.0 & 7.17 & 1.35 & -222 & $6.17 \mathrm{E}+00$ & 0.30 & 1.03 & 2.10 & 0.038 & $2.42 \mathrm{E}+00$ & b.d.l. & b.d.l. & 0.24 & 7.6 & $7.70 \mathrm{E}+02$ & -6.80 & -35.0 & -6.51 & -3.3 & 0.11 \\
\hline 10 & $\begin{array}{l}\text { A. Draham } \\
4068489,471870\end{array}$ & $11 / 10 / 2006$ & 18.7 & 4.69 & 0.3 & 247 & 8.94E-01 & 0.02 & 0.37 & 0.30 & 0.008 & $1.09 \mathrm{E}+00$ & b.d.l. & 0.03 & 0.68 & 0.1 & $1.13 \mathrm{E}+02$ & n.d & n.d & -7.61 & -3.45 & -5.02 \\
\hline 11 & $\begin{array}{l}\text { H. Ourahnya } \\
\quad 4085870,491169\end{array}$ & $12 / 10 / 2006$ & 34.8 & 6.42 & 2.62 & 30 & $1.33 \mathrm{E}+01$ & 0.51 & 1.37 & 5.48 & 0.002 & $1.44 \mathrm{E}+01$ & b.d.l. & b.d.l. & 0.11 & 5.8 & $1.32 \mathrm{E}+03$ & -6.83 & -35.8 & -5.43 & -3.36 & -0.4 \\
\hline 12 & $\begin{array}{l}\text { H. Nefza } \\
\quad 4092218,507232\end{array}$ & $11 / 10 / 2006$ & 33.7 & 6.51 & 3.16 & -110 & $9.72 \mathrm{E}+00$ & 0.18 & 1.63 & 10.3 & 0.039 & $1.47 \mathrm{E}+01$ & 0.15 & b.d.l. & 0.46 & 8.1 & $1.51 \mathrm{E}+03$ & -5.79 & -30.0 & -5.57 & -2.55 & 0.06 \\
\hline $13 a$ & $\begin{array}{l}\text { H. Kef-Ettout } \\
\quad 4084390,509626\end{array}$ & $11 / 10 / 2006$ & 40.8 & 6.31 & 10.5 & -110 & $5.29 \mathrm{E}+01$ & 0.85 & 3.29 & 21.0 & 0.150 & $7.13 \mathrm{E}+01$ & b.d.l. & 4.54 & 0.12 & 7.1 & $4.96 \mathrm{E}+03$ & -6.09 & -33.1 & -4.24 & -3.16 & 0.03 \\
\hline $13 \mathrm{~b}$ & $\begin{array}{l}\text { H. Kef-Ettout } \\
\quad 4084390,509626\end{array}$ & 29/09/2009 & 39.0 & n.d & 8.3 & n.d & n.d & n.d & n.d & n.d & n.d & n.d & n.d & n.d & n.d & n.d & n.d & -6.08 & -36.0 & n.d & n.d & n.d \\
\hline 14 & $\begin{array}{l}\text { A. El-Hammam (Marwa) } \\
4086086,536748\end{array}$ & 28/09/2009 & 22.4 & 7.16 & 0.72 & 240 & $1.71 \mathrm{E}+00$ & 0.09 & 0.61 & 5.03 & 0.016 & $2.66 \mathrm{E}+00$ & b.d.l. & 0.09 & 0.79 & 4.0 & $5.33 \mathrm{E}+02$ & -6.39 & -32.7 & -7.01 & -1.89 & 0.02 \\
\hline $15 a$ & $\begin{array}{l}\text { H. Sidi Abdelkader } \\
4110174,561458\end{array}$ & 09/10/2006 & 42.3 & 6.27 & 25.7 & -112 & $1.38 \mathrm{E}+02$ & 2.04 & 18.5 & 53.9 & 0.002 & $1.54 \mathrm{E}+02$ & b.d.l. & 0.72 & 48.3 & 5.9 & $1.27 \mathrm{E}+04$ & -4.23 & -21.8 & -3.58 & -0.51 & 0.14 \\
\hline $15 \mathrm{~b}$ & $\begin{array}{r}\text { H. Sidi Abdelkader } \\
4110174,561458\end{array}$ & $27 / 09 / 2009$ & 43.6 & 6.22 & 19.5 & n.d & n.d & n.d & n.d & n.d & n.d & n.d & n.d & n.d & n.d & n.d & n.d & -4.29 & -23.2 & n.d & n.d & n.d \\
\hline 16 & $\begin{array}{l}\text { H. Sidi Ben Abbès } \\
\text { 4110189, } 561473\end{array}$ & $27 / 09 / 2009$ & 42.0 & 6.30 & 27.2 & 33 & $1.39 \mathrm{E}+02$ & 1.35 & 18.3 & 55.9 & 0.000 & $1.61 \mathrm{E}+02$ & 0.11 & b.d.l. & 46.2 & 5.8 & $1.29 \mathrm{E}+04$ & -4.11 & -15.6 & -3.56 & -0.04 & 0.07 \\
\hline 17 & $\begin{array}{l}\text { H. Echfa (Ichkeul) } \\
4110204,561443\end{array}$ & $27 / 09 / 2009$ & 42.1 & 6.23 & 20.8 & n.d & n.d & n.d & n.d & n.d & n.d & n.d & b.d.l. & b.d.l. & n.d & n.d & n.d & -4.27 & -23.1 & n.d & n.d & n.d \\
\hline $18 a$ & $\begin{array}{l}\text { A. El-Hammam (Utique) } \\
4102028,594523\end{array}$ & 09/10/2006 & 38.6 & 7.21 & 2.73 & -60 & $1.39 \mathrm{E}+01$ & 0.33 & 3.02 & 3.38 & 0.039 & $1.24 \mathrm{E}+01$ & b.d.l. & 0.23 & 1.73 & 8.1 & $1.47 \mathrm{E}+03$ & -5.67 & -31.4 & -5.49 & -2.42 & 0.36 \\
\hline $18 \mathrm{~b}$ & $\begin{array}{l}\text { A. El-Hammam (Utique) } \\
4102028,594523\end{array}$ & $27 / 09 / 2009$ & 35.6 & 7.50 & 3.6 & 52 & $2.02 \mathrm{E}+01$ & 0.28 & 3.62 & 4.84 & 0.028 & $2.02 \mathrm{E}+01$ & 0.03 & 0.05 & 2.46 & 6.1 & $1.83 \mathrm{E}+03$ & -5.59 & -31.5 & -5.15 & -1.68 & 0.55 \\
\hline 22 & $\begin{array}{l}\text { A. Jebel Oust } \\
4043919,595741\end{array}$ & 03/10/2009 & 53.1 & 6.34 & 43.3 & n.d & $2.23 E+02$ & 3.74 & 17.3 & 51.8 & n.d. & $2.19 \mathrm{E}+02$ & b.d.l. & b.d.l. & 55.7 & 9.2 & $1.75 \mathrm{E}+04$ & -6.07 & -44.8 & -3.73 & -0.75 & 0.61 \\
\hline
\end{tabular}




\begin{tabular}{|c|c|c|c|c|c|c|c|c|c|c|c|c|c|c|c|c|c|c|c|c|c|c|}
\hline 27 & $\begin{array}{l}\text { F. Sahnoun (Jannet) } \\
3944800,560863\end{array}$ & 17/10/2006 & 23.2 & 7.54 & 0.54 & 45 & $1.11 \mathrm{E}+00$ & 0.09 & 1.58 & 2.29 & 0.030 & $7.20 \mathrm{E}-01$ & 0.01 & 0.07 & 0.62 & 3.7 & $3.81 \mathrm{E}+02$ & -6.40 & -39.1 & -7.73 & -2.79 & 0.1 \\
\hline 29 & $\begin{array}{l}\text { A. Maamar (H. Sollah) } \\
\text { 3918256, } 556021\end{array}$ & $17 / 10 / 2006$ & 34.9 & 6.26 & 59.3 & -353 & 3.13E + 02 & 8.33 & 27.3 & 67.3 & 0.002 & $4.33 \mathrm{E}+02$ & 2.00 & b.d.l. & 32.4 & 7.5 & $2.68 \mathrm{E}+04$ & -6.62 & -45.0 & -2.79 & -22.11 & -0.42 \\
\hline 30 & $\begin{array}{l}\text { A. Chenama (A. Mouailha) } \\
\text { 3916942, } 549495\end{array}$ & 30/09/2009 & 29.1 & 6.32 & 25.7 & -225 & $1.85 \mathrm{E}+02$ & 3.16 & 15.5 & 43.7 & n.d. & $2.09 \mathrm{E}+02$ & 0.14 & b.d.l. & 29.3 & 6.6 & $1.47 \mathrm{E}+04$ & -7.45 & -38.9 & -3.29 & -6.4 & -0.5 \\
\hline 31 & $\begin{array}{l}\text { A. Mizeb (Safia) } \\
\quad 3973336,489036\end{array}$ & 30/09/2009 & 16.1 & 7.44 & 0.43 & 95 & $5.48 \mathrm{E}-01$ & 0.02 & 1.08 & 3.73 & 0.021 & $5.90 \mathrm{E}-01$ & b.d.l. & 0.31 & 0.54 & 4.0 & $4.09 \mathrm{E}+02$ & -7.84 & -49.1 & -8.13 & -2.13 & 0.1 \\
\hline 32 & $\begin{array}{l}\text { A. Thala } \\
3935399,470659\end{array}$ & 13/10/2006 & 20.0 & 7.45 & 0.89 & 77 & $1.07 E+00$ & 0.07 & 2.53 & 4.97 & 0.051 & $1.30 \mathrm{E}+00$ & b.d.l. & 0.89 & 1.94 & 4.6 & $6.35 \mathrm{E}+02$ & -6.65 & -45.4 & -7.51 & -2.06 & 0.34 \\
\hline 33 & $\begin{array}{l}\text { F. Sidi Boulaaba } \\
3900858,477238\end{array}$ & 13/10/2006 & 47.2 & 6.84 & 4.08 & -155 & $1.22 \mathrm{E}+01$ & 0.43 & 5.34 & 10.8 & 0.054 & $8.14 \mathrm{E}+00$ & 0.03 & b.d.l. & 16.6 & 3.4 & $1.88 \mathrm{E}+03$ & -8.26 & -56.7 & -5.75 & -1.05 & 0.18 \\
\hline 34 & $\begin{array}{l}\text { F. Sidi Ahmed Zarouk Z1 } \\
\text { 3810949, } 476376\end{array}$ & $13 / 10 / 2006$ & 29.1 & 6.51 & 20.4 & -275 & $2.06 \mathrm{E}+02$ & 4.06 & 16.1 & 36.3 & 0.002 & $2.13 \mathrm{E}+02$ & 1.31 & b.d.l. & 39.0 & 6.8 & $1.58 \mathrm{E}+04$ & -7.14 & -49.8 & -3.25 & -12.17 & -0.09 \\
\hline 35 & $\begin{array}{l}\text { F. Sidi Ahmed Zarouk Z3 } \\
\text { 3811485, } 476264\end{array}$ & 13/10/2006 & 28.7 & 6.65 & 21.3 & 114 & $1.52 \mathrm{E}+02$ & 3.29 & 14.9 & 32.3 & 0.002 & $1.59 \mathrm{E}+02$ & 0.99 & 0.81 & 38.8 & 6.2 & $1.24 \mathrm{E}+04$ & -7.29 & -48.8 & -3.49 & -0.76 & 0.15 \\
\hline \multicolumn{23}{|c|}{ Eastern samples } \\
\hline 19 & $\begin{array}{l}\text { A. El-Ariane } \\
4065274,619429\end{array}$ & 08/10/2006 & 47.7 & 6.07 & 32.8 & 206 & $1.70 \mathrm{E}+02$ & 3.70 & 12.7 & 50.2 & 0.241 & $2.00 \mathrm{E}+02$ & b.d.l. & b.d.l. & 25.3 & 9.9 & $1.42 \mathrm{E}+04$ & -5.66 & -33.0 & -3.39 & -0.83 & 0.19 \\
\hline 20 & $\begin{array}{l}\text { A. Echfa (Korbous) } \\
4075691,639811\end{array}$ & 09/10/2006 & 52.7 & 6.57 & 26.7 & 90 & $1.10 \mathrm{E}+02$ & 2.10 & 14.4 & 39.4 & 0.002 & $1.34 \mathrm{E}+02$ & 0.54 & b.d.l. & 30.8 & 7.9 & $1.03 \mathrm{E}+04$ & -5.01 & -26.2 & n.d & n.d & n.d \\
\hline 21 & $\begin{array}{l}\text { A. El-Atrous (Korbous) } \\
4076906,639935\end{array}$ & 08/10/2006 & 58.0 & 6.30 & 29.6 & -200 & $1.11 \mathrm{E}+02$ & 1.89 & 13.8 & 38.9 & 0.002 & $1.37 \mathrm{E}+02$ & 0.99 & b.d.l. & 33.2 & 5.3 & $1.04 \mathrm{E}+04$ & -4.99 & -26.9 & -3.72 & -0.86 & 0.2 \\
\hline 23 & $\begin{array}{l}\text { H. Jedidi } \\
\text { 4028517, } 624445\end{array}$ & 18/10/2006 & 53.3 & 6.66 & 4.49 & 35 & $2.32 \mathrm{E}+02$ & 4.19 & 8.17 & 34.0 & 0.002 & $2.38 \mathrm{E}+02$ & b.d.l. & b.d.l. & 33.9 & 7.0 & $1.68 \mathrm{E}+04$ & -6.04 & -39.0 & -3.2 & -0.87 & 0.52 \\
\hline 24 & $\begin{array}{l}\text { H. Zriba } \\
\quad 4022550,607773\end{array}$ & 19/10/2006 & 44.1 & 6.57 & 13.1 & 154 & $4.22 \mathrm{E}+01$ & 0.95 & 11.3 & 33.2 & 0.208 & $4.97 \mathrm{E}+01$ & b.d.l. & b.d.l. & 40.9 & 4.1 & $5.79 \mathrm{E}+03$ & -5.66 & -36.1 & -4.51 & -0.52 & 0.25 \\
\hline 25 & $\begin{array}{l}\text { A. Garci } \\
4000811,613123\end{array}$ & $17 / 10 / 2006$ & 23.1 & 6.63 & 3.22 & 30 & $1.55 \mathrm{E}+01$ & 0.42 & 4.64 & 7.17 & 0.102 & $9.96 \mathrm{E}+00$ & b.d.l. & 0.12 & 2.94 & 16.8 & $2.10 \mathrm{E}+03$ & -5.48 & -36.7 & -5.53 & -1.97 & 0.14 \\
\hline 26 & $\begin{array}{l}\text { F. Sidi Abdelhamid } \\
3962682,649531\end{array}$ & 03/10/2009 & 22.2 & 7.39 & 12.1 & -205 & $1.04 \mathrm{E}+02$ & 0.59 & 12.4 & 12.9 & n.d. & $1.06 \mathrm{E}+02$ & b.d.l. & 0.25 & 24.6 & 3.0 & $7.97 \mathrm{E}+03$ & -5.59 & -37.1 & -3.78 & -0.68 & 0.11 \\
\hline 28 & $\begin{array}{l}\text { F. Oued Kharrouba (Sabrine) } \\
3942387,583388\end{array}$ & 30/09/2009 & 25.3 & 7.64 & 0.54 & 40 & $2.09 \mathrm{E}+00$ & 0.50 & 0.94 & 1.96 & 0.081 & $9.51 \mathrm{E}-01$ & b.d.l. & 0.22 & 0.55 & 3.8 & $4.26 \mathrm{E}+02$ & -5.27 & -30.2 & -7.36 & -2.38 & 0.14 \\
\hline 41 & $\begin{array}{l}\text { F. Zarat } \\
\quad 3724907,623871\end{array}$ & 15/10/2006 & 31.5 & 7.27 & 4.48 & -27 & $1.39 \mathrm{E}+01$ & 0.39 & 8.49 & 13.2 & 0.124 & $1.33 \mathrm{E}+01$ & 0.06 & 0.37 & 19.2 & 2.5 & $2.28 \mathrm{E}+03$ & -6.95 & -47.5 & -5.48 & -0.98 & 0.31 \\
\hline 42 & $\begin{array}{l}\text { F. Alaya } \\
3722884,622751\end{array}$ & $15 / 10 / 2006$ & 31.6 & 7.14 & 4.28 & 69 & $1.41 \mathrm{E}+01$ & 0.36 & 8.60 & 13.5 & 0.122 & $1.37 \mathrm{E}+01$ & 0.06 & 0.39 & 19.5 & 2.5 & $2.32 \mathrm{E}+03$ & -6.99 & -47.1 & -5.46 & -0.97 & 0.19 \\
\hline 43 & $\begin{array}{l}\text { F. Les Sirènes } \\
\quad 3748188,683623\end{array}$ & 16/10/2006 & 30.4 & 7.42 & 12.5 & -180 & $7.39 \mathrm{E}+01$ & 0.95 & 11.3 & 14.4 & 0.002 & $6.89 \mathrm{E}+01$ & 0.57 & b.d.l. & 28.8 & 2.6 & $6.19 \mathrm{E}+03$ & -6.33 & -44.0 & -4.11 & -1 & 0.34 \\
\hline \multicolumn{23}{|c|}{ samples } \\
\hline 36 & $\begin{array}{l}\text { H. Mahassen } \\
\text { 3763954, } 429972\end{array}$ & $14 / 10 / 2006$ & 63.0 & 7.07 & 6.08 & -190 & $1.22 \mathrm{E}+01$ & 2.00 & 2.83 & 15.26 & 0.002 & $1.20 \mathrm{E}+01$ & b.d.l. & b.d.l. & 19.33 & 2.7 & $2.22 \mathrm{E}+03$ & -7.50 & -53.4 & -5.61 & -0.83 & 0.63 \\
\hline 37 & $\begin{array}{l}\text { H. Ras El-Aïn } \\
\quad 3729020,498612\end{array}$ & 14/10/2006 & 49.3 & 8.00 & 5.72 & -20 & $1.55 \mathrm{E}+01$ & 0.82 & 4.68 & 11.62 & 0.002 & $1.71 \mathrm{E}+01$ & 0.12 & b.d.l. & 14.95 & 2.1 & $2.14 \mathrm{E}+03$ & -8.14 & -60.2 & -5.34 & -1.09 & 1.12 \\
\hline 38 & $\begin{array}{l}\text { F. Ras El-Aïn } \\
\quad 3729020,498612\end{array}$ & 15/10/2006 & 64.0 & 7.28 & 6.77 & -243 & $1.32 \mathrm{E}+01$ & 0.95 & 4.91 & 11.78 & 0.002 & $1.67 \mathrm{E}+01$ & 0.10 & b.d.l. & 14.50 & 2.2 & $2.07 \mathrm{E}+03$ & -8.48 & -61.6 & -5.43 & -1.08 & 0.65 \\
\hline 39 & $\begin{array}{l}\text { F. El-Hamma F2 } \\
\text { 3753067, } 562663\end{array}$ & 15/10/2006 & 67.3 & 6.73 & 7.22 & -204 & $1.37 \mathrm{E}+01$ & 1.10 & 5.20 & 17.25 & 0.002 & $1.75 \mathrm{E}+01$ & b.d.l. & 0.06 & 19.08 & 2.5 & $2.46 \mathrm{E}+03$ & -8.35 & -61.4 & -5.41 & -0.85 & 0.35 \\
\hline 40 & $\begin{array}{l}\text { F. El-Hamma F9 } \\
\text { 3753067, } 562663\end{array}$ & 15/10/2006 & 63.6 & 6.81 & 8.52 & -197 & $2.01 \mathrm{E}+01$ & 1.00 & 5.45 & 19.18 & 0.002 & $2.27 \mathrm{E}+01$ & 0.16 & b.d.l. & 19.88 & 2.0 & $2.85 \mathrm{E}+03$ & -8.28 & -59.6 & -5.14 & -0.84 & 0.3 \\
\hline
\end{tabular}

A stands for Aïn, the arab word for spring, $\mathrm{F}$ for drilled wells, and $\mathrm{H}$ for hammam.

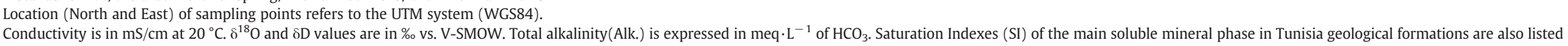
Conductivity is in $\mathrm{mS} / \mathrm{cm}$ at $20^{\circ} \mathrm{C} . \delta^{18} \mathrm{O}$ and $\delta \mathrm{D}$ values are in \% vs.
(calculated with the PHREEQC code, Parkhurst and Appelo, 1999).

(calculated with the PHREEQC code, Parkhurst and App
n.d. = not determined; b.d. = below detection limit. 
Table 2

Dissolved gas and carbon and helium isotopes composition of Tunisian groundwaters.

\begin{tabular}{|c|c|c|c|c|c|c|c|c|c|c|c|c|c|c|c|c|c|c|c|c|c|}
\hline \multirow[t]{2}{*}{ Sample } & \multirow[t]{2}{*}{ Site name } & \multirow{2}{*}{$\frac{\mathrm{P}_{\text {tot }}}{\mathrm{atm} .}$} & \multirow{2}{*}{$\frac{\mathrm{N}_{2}}{\% \mathrm{vol}}$} & \multirow{2}{*}{$\frac{\mathrm{O}_{2}}{\% \mathrm{vol}}$} & \multirow{2}{*}{$\frac{\mathrm{CO}_{2}}{\% \mathrm{vol}}$} & \multirow{2}{*}{$\frac{\mathrm{H}_{2}}{\% \text { vol }}$} & \multirow{2}{*}{$\begin{array}{l}\mathrm{CO} \\
\% \mathrm{vol}\end{array}$} & \multirow{2}{*}{$\frac{\mathrm{CH}_{4}}{\% \mathrm{vol}}$} & \multirow{2}{*}{$\frac{\text { TDIC }}{\mathrm{mol} / \mathrm{l}}$} & \multirow{2}{*}{$\frac{\delta^{13} C_{\text {TDIC }}}{\%}$} & \multirow{2}{*}{$\frac{\delta^{13} \mathrm{C}_{\mathrm{CO} 2}}{\%}$} & \multirow{2}{*}{$\frac{{ }^{4} \mathrm{He}}{\text { mes (1) }}$} & \multirow{2}{*}{$\frac{{ }^{4} \mathrm{He}}{\text { mes (2) }}$} & \multirow{2}{*}{$\frac{{ }^{4} \mathrm{He} /{ }^{20} \mathrm{Ne}}{\text { mes (1) }}$} & \multirow{2}{*}{$\frac{{ }^{4} \mathrm{He} /{ }^{20} \mathrm{Ne}}{\mathrm{mes}(2)}$} & \multirow{2}{*}{$\frac{\mathrm{R} / \mathrm{R}_{\mathrm{a}}}{\mathrm{mes}(1)}$} & \multirow{2}{*}{$\frac{\mathrm{R} / \mathrm{R}_{\mathrm{a}}}{\text { mes (2) }}$} & \multirow{2}{*}{$\frac{{ }^{4} \mathrm{He}}{\mathrm{xs}(1)}$} & \multirow{2}{*}{$\frac{{ }^{4} \mathrm{He}}{\mathrm{xs}(2)}$} & \multirow{2}{*}{$\frac{\mathrm{R} / \mathrm{R}_{\mathrm{a}}}{\mathrm{xs}(1)}$} & \multirow{2}{*}{$\frac{\mathrm{R} / \mathrm{R}_{\mathrm{a}}}{\mathrm{xs}(2)}$} \\
\hline & & & & & & & & & & & & & & & & & & & & & \\
\hline \multicolumn{22}{|c|}{ Atlasic samples } \\
\hline 1 & H. Mallègue & 2.38 & 85.80 & 9.59 & 4.60 & $2.7 \mathrm{E}-03$ & $8.2 \mathrm{E}-04$ & 7.4E-03 & $1.1 \mathrm{E}-02$ & -2.61 & -7.02 & 67.5 & n.d & 6.937 & n.d & 0.09 & n.d & 65 & n.d & 0.055 & n.d \\
\hline 2 & H. Bezzaz & n.d & n.d & n.d & n.d & n.d & n.d & n.d & n.d & n.d & n.d & 8.04 & 8.71 & 0.975 & 1.071 & 0.42 & 0.395 & 5.88 & 6.58 & 0.213 & 0.204 \\
\hline 3 & H. Ouchtata & 1.08 & 82.98 & 0.06 & 9.98 & b.d.l. & $4.1 \mathrm{E}-05$ & $7.0 \mathrm{E}+00$ & $1.4 \mathrm{E}-02$ & -0.56 & -5.19 & 35.2 & n.d & 2.146 & n.d & 0.137 & n.d & 30.9 & n.d & 0.019 & n.d \\
\hline 4 & H. Ali Daoua & 1.34 & 80.92 & 3.70 & 15.36 & $2.8 \mathrm{E}-03$ & $3.1 \mathrm{E}-04$ & $1.8 \mathrm{E}-02$ & $1.6 \mathrm{E}-02$ & -0.41 & -4.30 & 28.5 & 29.8 & 2.929 & 3.307 & 0.179 & 0.174 & 26 & 27.4 & 0.1 & 0.104 \\
\hline $5 a$ & H. Biadha & 1.28 & 59.68 & 0.07 & 40.24 & $5.2 \mathrm{E}-03$ & $1.7 \mathrm{E}-04$ & $9.6 \mathrm{E}-04$ & $3.9 \mathrm{E}-02$ & 2.89 & -0.93 & 2.73 & 2.88 & 1.167 & 3.789 & 0.424 & 0.247 & 2.12 & 2.68 & 0.262 & 0.191 \\
\hline $5 b$ & H. Biadha & n.d & n.d & n.d & n.d & n.d & n.d & n.d & n.d & n.d & n.d & 2.5 & 2.5 & 4.310 & 1.678 & 0.229 & 0.349 & 2.35 & 2.11 & 0.179 & 0.231 \\
\hline $6 a$ & H. Salaya & 1.68 & 85.47 & 9.43 & 5.08 & $3.1 \mathrm{E}-03$ & b.d.l. & $2.7 \mathrm{E}-04$ & $1.2 \mathrm{E}-02$ & -0.48 & -5.05 & 173.9 & n.d & 13.336 & n.d & 0.121 & n.d & 170 & n.d & 0.104 & n.d \\
\hline $6 \mathrm{~b}$ & H. Salaya & 1.11 & 90.68 & 0.11 & 9.19 & b.d.l. & $2.1 \mathrm{E}-04$ & $1.1 \mathrm{E}-02$ & $1.2 \mathrm{E}-02$ & -6.26 & -10.78 & 107 & 119 & 7.698 & 7.933 & 0.135 & 0.134 & 103 & 115 & 0.105 & 0.105 \\
\hline $7 a$ & H. Es-Salhine & 3.01 & 95.62 & 1.60 & 2.77 & $8.9 \mathrm{E}-04$ & $6.6 \mathrm{E}-05$ & $1.9 \mathrm{E}-03$ & $5.1 \mathrm{E}-03$ & -5.67 & -7.29 & 13.7 & n.d & 1.745 & n.d & 0.368 & n.d & 11.6 & n.d & 0.26 & n.d \\
\hline $7 \mathrm{~b}$ & H. Es-Salhine & 0.91 & 72.44 & 0.37 & 13.86 & n.d & b.d.l. & $1.3 \mathrm{E}+01$ & $5.6 \mathrm{E}-03$ & -10.09 & -11.45 & 15.7 & 16.4 & 2.096 & 2.207 & 0.35 & 0.335 & 13.7 & 14.5 & 0.259 & 0.248 \\
\hline 8 & H. Bourguiba ("populaire") & 2.10 & 93.00 & 2.47 & 4.52 & $1.2 \mathrm{E}-03$ & $3.2 \mathrm{E}-04$ & $3.1 \mathrm{E}-03$ & $1.1 \mathrm{E}-02$ & -3.10 & -7.32 & n.d & n.d & n.d & n.d & n.d & n.d & n.d & n.d & n.d & n.d \\
\hline 9 & H. Bourguiba ("basse") & 1.45 & 94.52 & 1.77 & 3.69 & $1.2 \mathrm{E}-03$ & b.d.l. & $1.3 \mathrm{E}-02$ & $9.1 \mathrm{E}-03$ & -4.54 & -10.13 & 62.9 & 58 & 3.709 & 3.418 & 0.204 & 0.2 & 58.5 & 53.6 & 0.144 & 0.135 \\
\hline 10 & A Draham & n.d & n.d & n.d & n.d & n.d & $n d$ & $n d$ & n.d & n.d & n.d & n.d & $n d$ & n.d & n.d & n.d & n.d & n.d & n.d & n.d & n.d \\
\hline 11 & H. Ourahnya & n.d & n.d & n.d & n.d & n.d & 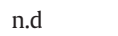 & & n.d & n.d & n.d & 36.8 & 37.9 & 2.564 & 2.411 & 0.147 & 0.152 & 33 & 33.8 & 0.052 & 0.05 \\
\hline 12 & H. Nefza & 1.93 & 86.83 & 7.07 & 6.10 & $2.5 \mathrm{E}-03$ & $5.1 \mathrm{E}-05$ & $1.8 \mathrm{E}-04$ & $1.1 \mathrm{E}-02$ & -4.52 & -9.14 & 51.6 & n.d & 2.571 & n.d & 0.209 & n.d & 46.3 & n.d & 0.121 & n.d \\
\hline $13 a$ & H. Kef-Ett & 1.70 & 92.51 & 0.11 & 7.38 & $1.6 \mathrm{E}-03$ & $0.0 \mathrm{E}+00$ & $5.8 \mathrm{E}-04$ & $1.0 \mathrm{E}-02$ & -3.48 & -7.45 & 43.9 & 77.5 & 2.698 & 3.827 & 0116 & 0.123 & 39.6 & 72.2 & 0.023 & 0.06 \\
\hline $13 \mathrm{~b}$ & H. Kef-Ettout & n.d & n.d & n.d & n.d & n.d & n.d & n.d & n.d & n.d & n.d & 17.4 & n.d & 1.289 & n.d & 0.227 & n.d & 13.8 & n.d & 0.033 & n.d \\
\hline 14 & A. El-Hamman & 1.61 & 83.59 & 15.34 & 1.06 & b.d.l. & $4.1 \mathrm{E}-03$ & $3.6 \mathrm{E}-04$ & $4.6 \mathrm{E}-03$ & -10.20 & -17.15 & 18.9 & 18.2 & 0.325 & 0.329 & 0.872 & 0.858 & 3.64 & 3.74 & 0.399 & 0.367 \\
\hline $15 a$ & H. Sidi Abdelkader & 1.42 & 89.38 & 2.96 & 7.66 & b.d.l. & b.d.l. & $1.0 \mathrm{E}-03$ & $8.4 \mathrm{E}-03$ & -2.44 & -6.28 & 37.4 & 59.1 & 0.822 & 5.175 & 0.651 & 0.427 & 25.5 & 56.1 & 0.495 & 0.397 \\
\hline $15 b$ & H. Sidi Abdelkader & n.d & n.d & n.d & n.d & n.d & n.d & n.d & n.d & n.d & n.d & 113 & n.d & 8.188 & n.d & 0.417 & n.d & 110 & n.d & 0.399 & n.d \\
\hline 16 & H. Sidi Ben Abbès & 1.15 & 86.91 & 0.07 & 12.99 & b.d.l. & b.d.l. & $2.1 \mathrm{E}-02$ & $9.3 \mathrm{E}-03$ & -9.67 & -12.99 & 100 & 112 & 0.877 & 7.619 & 0.603 & 0.425 & 70.2 & 108 & 0.441 & 0.405 \\
\hline 17 & H. Echfa (Ichkeul) & n.d & n.d & n.d & n.d & n.d & n.d & n.d & n.d & n.d & n.d & 86.4 & 108 & 7.927 & 7.714 & 0.416 & 0.428 & 83.5 & 105 & 0.397 & 0.408 \\
\hline $18 \mathrm{a}$ & A. El-Hammam (Ut & 1.26 & 85.38 & 13.37 & 1.25 & $2.1 \mathrm{E}-03$ & b.d.l. & $2.0 \mathrm{E}-04$ & $8.5 \mathrm{E}-03$ & -7.75 & -13.78 & 7.57 & 9.07 & 2.273 & 2.296 & 0.547 & 0.558 & 3.71 & 4.42 & 0.093 & 0.107 \\
\hline $18 \mathrm{~b}$ & A. El-Hammam (Utique) & 1.11 & 91.69 & 5.80 & 2.51 & b.d.l. & 7.1E-05 & $1.2 \mathrm{E}-04$ & $6.8 \mathrm{E}-03$ & -11.23 & -17.10 & 10.1 & 10 & 0.526 & 0.549 & 0.52 & 0.524 & 5.06 & 5.22 & 0.058 & 0.098 \\
\hline 22 & A. Jebel Oust & 1.66 & 80.59 & 8.89 & 10.50 & b.d.l. & $1.3 \mathrm{E}-04$ & $1.1 \mathrm{E}-03$ & $1.3 \mathrm{E}-02$ & -3.88 & -7.06 & 107 & 113 & 8.492 & 7.847 & 0.228 & 0.223 & 103 & 109 & 0.203 & 0.197 \\
\hline 27 & F. Sahnoun (Jannet) & 1.05 & 97.01 & 2.16 & 0.84 & b.d.l. & $6.4 \mathrm{E}-05$ & $5.2 \mathrm{E}-04$ & & & & 11.4 & 11.2 & 0.535 & 0.521 & 0.521 & 0.52 & 5.82 & 5.57 & 0.076 & 0.049 \\
\hline
\end{tabular}




$\begin{array}{ll}29 & \text { A. Maamar (H. Sollah) } \\ 30 & \text { A. Chenama (A. Mouailha) } \\ 31 & \text { A. Mizeb (Safia) } \\ 32 & \text { A. Thala } \\ 33 & \text { F. Sidi Boulaaba } \\ 34 & \text { F. Sidi Ahmed Zarouk Z1 } \\ 35 & \text { F. Sidi Ahmed Zarouk Z3 }\end{array}$

\begin{tabular}{|c|c|c|c|c|c|c|c|c|c|c|c|c|c|c|c|c|c|c|c|}
\hline & 83.97 & 1.65 & 3.26 & b.d.l. & $\mathrm{D}$ & $8 \mathrm{E}-01$ & 1.2 & $-\varepsilon$ & -1 & 4 & .6 & 73 & 73.121 & 1 & 1 & 2330 & 2750 & 0.018 & 0.018 \\
\hline & n.d & n.d & n.d & n.d & n.d & n.d & n.d & n.d & n.d & 3260 & 40 & 60.708 & 80.276 & 0 & 6 & 3250 & 4050 & 0.021 & 0.023 \\
\hline & 96.04 & 2.96 & 1.00 & b.d.l. & b.d.l. & 75 & n.d & n.d & nd & 5.12 & 5.2 & 0.265 & 20 & 1.034 & 07 & d & nd & n.d & n.d \\
\hline & n.d & n.d & 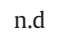 & n.d & & & t.d & & & & & & & & .व & 1. & & n.d & 1.व \\
\hline & 95.41 & .27 & 1.31 & 3 & & & 03 & & & & & & & & & & & 0.022 & 0.019 \\
\hline & 95.44 & 0.64 & 3.62 & $1.4 \mathrm{E}-03$ & 1E-05 & 3E-02 & $3 \mathrm{E}-03$ & -3.88 & -9.05 & 4723 & 4662 & 187 & 16.434 & $0 . c$ & 1 & 13 & 4652 & 0.019 & 019 \\
\hline & 93.40 & 3.08 & 3.40 & $6.5 \mathrm{E}-03$ & $2.5 \mathrm{E}-04$ & $1.6 \mathrm{E}-04$ & 7.9E-03 & -4.31 & -9.91 & 1777 & 1452 & 75.778 & 69.774 & 0.023 & 0.021 & 1770 & 1450 & 0.019 & 0.018 \\
\hline
\end{tabular}

Eastern samples

\begin{tabular}{|c|c|c|c|c|c|c|c|c|c|c|c|c|c|c|c|c|c|c|c|c|c|}
\hline \\
\hline 19 & A. El-Ariane & 1.92 & 73.20 & 6.84 & 19.92 & 4.1E-03 & $3.0 \mathrm{E}-04$ & $5.7 \mathrm{E}-03$ & $1.8 \mathrm{E}-02$ & -0.30 & -2.76 & 344 & 527 & 43.544 & 35.705 & 0.287 & 0.289 & 34.2 & 52.3 & 0.283 & 0.284 \\
\hline 20 & A. Echfa (Korbous) & 1.09 & 77.35 & 6.91 & 15.73 & $2.1 \mathrm{E}-03$ & b.d.l. & $2.0 \mathrm{E}-03$ & $1.1 \mathrm{E}-02$ & -0.38 & -3.44 & 288.8 & 307.1 & 22.902 & 30.466 & 0.267 & 0.263 & 385 & 304 & 0.261 & 0.257 \\
\hline 21 & A. El-Atrous (Korbous) & 2.19 & 73.25 & 12.74 & 13.98 & $6.9 \mathrm{E}-03$ & $7.1 \mathrm{E}-04$ & $7.0 \mathrm{E}-04$ & $1.1 \mathrm{E}-02$ & -0.89 & -2.44 & 441.8 & 442.7 & 54.678 & 54.120 & 0.266 & 0.266 & 440 & 441 & 0.263 & 0.262 \\
\hline 23 & H. Jedidi & 1.72 & 88.45 & 5.39 & 6.09 & $7.8 \mathrm{E}-03$ & $4.8 \mathrm{E}-02$ & $1.7 \mathrm{E}-02$ & $9.0 \mathrm{E}-03$ & 0.30 & -3.12 & 20.1 & 22.9 & 1.811 & 1.800 & 0.712 & 0.708 & 17.2 & 19.6 & 0.665 & 0.661 \\
\hline 24 & H. Zriba & 1.04 & 93.06 & 1.17 & 5.76 & b.d.l. & $1.2 \mathrm{E}-04$ & $7.1 \mathrm{E}-03$ & $5.4 \mathrm{E}-03$ & -4.56 & -8.61 & 54 & 33.4 & 3.902 & 3.337 & 0.631 & 0.621 & 50.4 & 30.8 & 0.605 & 0.59 \\
\hline 25 & A. Garci & 1.15 & 78.74 & 1.81 & 19.42 & b.d.l. & $1.3 \mathrm{E}-04$ & $1.5 \mathrm{E}-02$ & $2.5 \mathrm{E}-02$ & -0.56 & -5.70 & 141 & 136.9 & 9.704 & 8.930 & 2.392 & 2.388 & 137 & 132.9 & 2.431 & 2.431 \\
\hline 26 & F. Sidi Abdelhamid & 1.52 & 99.19 & 0.15 & 0.51 & b.d.l. & b.d.l. & $6.5 \mathrm{E}-02$ & $3.3 \mathrm{E}-03$ & -5.96 & -13.39 & 3800 & n.d & 62.500 & n.d & 0.314 & n.d & 3790 & n.d & 0.311 & n.d \\
\hline 28 & F. Oued Kharrouba (Sabrine) & 0.91 & 98.68 & 0.08 & 1.24 & b.d.l. & b.d.l. & $3.5 \mathrm{E}-04$ & $4.2 \mathrm{E}-03$ & -8.81 & -15.88 & 5.53 & 5.34 & 0.287 & 0.287 & 1.022 & 1.04 & n.d & n.c & n.d & n.d \\
\hline 41 & F. Zarat & 1.65 & 91.85 & 7.65 & 0.49 & b.d.l. & b.d.l. & $2.4 \mathrm{E}-04$ & $2.7 \mathrm{E}-03$ & -5.96 & -12.42 & 23.9 & n.d & 1.273 & n.d & 0.239 & n.d & 19 & n.d & 0.046 & n.d \\
\hline 42 & F. Alaya & 1.67 & 94.40 & 5.13 & 0.45 & b.d.l. & $9.7 \mathrm{E}-05$ & $1.7 \mathrm{E}-02$ & $2.7 \mathrm{E}-03$ & -6.26 & -12.75 & 11.3 & 16.6 & 0.556 & 0.846 & 0.503 & 0.335 & 5.98 & 11.5 & 0.074 & 0.044 \\
\hline 43 & F. Les Sirènes & 1.36 & 96.16 & 3.42 & 0.40 & b.d.l. & $2.0 \mathrm{E}-04$ & $1.5 \mathrm{E}-02$ & $2.8 \mathrm{E}-03$ & -10.09 & -16.90 & 651.1 & n.d & 21.893 & n.d & 0.121 & n.d & 643 & n.d & 0.111 & n.d \\
\hline
\end{tabular}

Saharan samples

\begin{tabular}{|c|c|c|c|c|c|c|c|c|c|c|c|c|c|c|c|c|c|c|c|c|c|}
\hline 36 & H. Mahassen & 3.31 & 91.82 & 5.99 & 1.87 & $1.5 \mathrm{E}-03$ & $2.3 \mathrm{E}-01$ & $9.2 \mathrm{E}-02$ & $3.7 \mathrm{E}-03$ & -7.79 & -10.11 & 115.9 & 101.8 & 8.273 & 7.442 & 0.042 & 0.045 & 112 & 98.2 & 0.011 & 0.011 \\
\hline 37 & H. Ras El-Aïn & 1.48 & 96.14 & 3.62 & 0.21 & b.d.l. & $1.8 \mathrm{E}-04$ & $2.3 \mathrm{E}-02$ & $2.2 \mathrm{E}-03$ & -10.83 & -15.80 & n.d & n.d & n.d & n.d & n.d & n.d & n.d & n.d & n.d & n.d \\
\hline 38 & F. Ras El-Aïn & 1.46 & 95.78 & 2.80 & 1.42 & b.d.l. & b.d.l. & $7.2 \mathrm{E}-04$ & $2.5 \mathrm{E}-03$ & -9.67 & -12.55 & 20.2 & 19.9 & 1.688 & 1.605 & 0.194 & 0.196 & 17.1 & 16.7 & 0.048 & 0.042 \\
\hline 39 & F. El-Hamma F2 & 2.59 & 96.88 & 1.72 & 1.29 & $1.4 \mathrm{E}-03$ & $1.6 \mathrm{E}-04$ & $1.0 \mathrm{E}-01$ & $3.0 \mathrm{E}-03$ & -10.20 & -12.59 & 404.8 & 268.2 & 13.435 & 12.907 & 0.036 & 0.035 & 397 & 263 & 0.017 & 0.016 \\
\hline 40 & F. El-Hamma F9 & 1.66 & 97.78 & 0.49 & 1.59 & b.d.l. & b.d.l. & $1.3 \mathrm{E}-01$ & 2.4E-03 & -11.23 & -13.94 & 162.1 & 196.3 & 6.049 & 6.541 & 0.053 & 0.051 & 155 & 188 & 0.011 & 0.012 \\
\hline
\end{tabular}

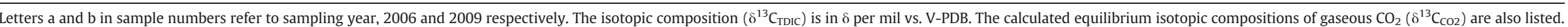

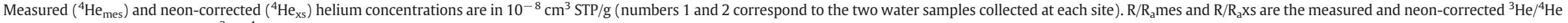
ratios scaled to the atmospheric ${ }^{3} \mathrm{He} /{ }^{4} \mathrm{He}$ value (Ra) 
uncertainty on measured ${ }^{3} \mathrm{He} /{ }^{4} \mathrm{He}$ ratios, calibrated against an atmospheric air standard, is better than $0.5 \%$. At each site, two helium samples were collected, which give consistent results (Table 2). Helium concentrations and isotopic ratios were corrected for possible air contamination using the measured ${ }^{20} \mathrm{Ne} /{ }^{4} \mathrm{He}$ ratios and assuming a pure atmospheric origin for ${ }^{20} \mathrm{Ne}$ (Hilton, 1996).

\section{Results}

\subsection{Chemistry of major ions}

The collected water samples are characterized by significant heterogeneity in their chemical-physical features (see Table 1). We identify both cold $\left(\mathrm{T}<25^{\circ} \mathrm{C}\right.$ ) and thermal ( $\mathrm{T}$ from 25 to $73.4^{\circ} \mathrm{C}$ ) groundwaters in our dataset. The waters range from dilute (with TDS, as low as $113 \mathrm{mg} \mathrm{L}^{-1}$; Total Dissolved Solid (TDS) content is the calculated sum of the major and minor ion species contents in $\mathrm{mg} \mathrm{L}^{-1}$ ) to saline waters, and show $\mathrm{pH}$ values from slightly acidic (e.g. sample 10) to nearly neutral (e.g. samples 27 and 28). Thermal waters are characterized by very high TDS values (up to $53,000 \mathrm{mg} \mathrm{L}^{-1}$ ), usually even greater than those typical of seawater. Overall, thermal waters display a weak but systematic positive correlation between salinity and $\mathrm{pH}$ values, suggesting that their saline burden reflects the temperature-dependent titration of the formerly acidic infiltrating meteoric waters by dissolving host rocks.

The collected water samples belong to all of the four water classification types proposed by Langelier and Ludwig (1942) (Fig. 2). More in the specific, cold waters flowing in the terrains of the Atlasic and Eastern domains have a prevalent bicarbonate earth-alkaline composition characteristic of shallow infiltrated meteoric waters in sedimentary environment. We note however that a few cold water samples, collected near the coast (e.g. sample 26), depart from the bicarbonate-type waters, and trend towards the compositional field of chloride-sulfate alkaline waters; this, when looked in tandem with increasing TDS values, supports some extent of seawater contribution to coastal shallow aquifers, as already reported in Tunisia for instance in the case of the Korba aquifer, Cap Bon (Kouzana et al., 2009, 2010) or the Sfax superficial aquifer (Trabelsi et al., 2005, 2007).

Thermal waters display a large spread of compositions. Samples collected in the Atlasic domain are the most heterogeneous. They have prevalent chloride-sulfate alkaline compositions, with $\mathrm{Na}$ as major cation species (Ca prevails only in samples 12 and 33, see Fig. 2); but also spread towards bicarbonate-alkaline compositions (as indicated by the arrow in Fig. 2) to occasionally chloride-sulfate earth alkaline compositions. Thermal groundwaters from the Eastern and Saharan domains, in turn, have more uniform compositions, and cluster in a relatively narrow area along the left segment of Fig. 2.

The contrasted compositional features of Tunisian groundwaters emerge even more evidently when the main anion $\left(\mathrm{Cl}, \mathrm{SO}_{4}\right.$ and $\left.\mathrm{HCO}_{3}\right)$ and cation $(\mathrm{Na}, \mathrm{Ca}$, and $\mathrm{Mg}+\mathrm{K}$ ) dissolved species are separately analyzed. Inspection of ternary diagrams (Fig. 3a \& b) demonstrates that:

(i) $\mathrm{Mg}$ and $\mathrm{K}$ are a relatively minor species in all Tunisian groundwaters; which, in terms of dissolved cations range from Ca-rich to Na-rich (Fig. 3a). The group of Na-rich waters includes all thermal water samples and most cold groundwaters;

(ii) Bicarbonate is the main anion species in most cold-groundwaters, consistent with the prevailingly meteoric/organic origin of dissolved salts (from atmospheric and soil $\mathrm{CO}_{2}$ ) in these poorly mineralized water samples (Fig. 3b). Only in high-TDS cold groundwaters, $\mathrm{Cl}$ prevails over $\mathrm{HCO}_{3}$;

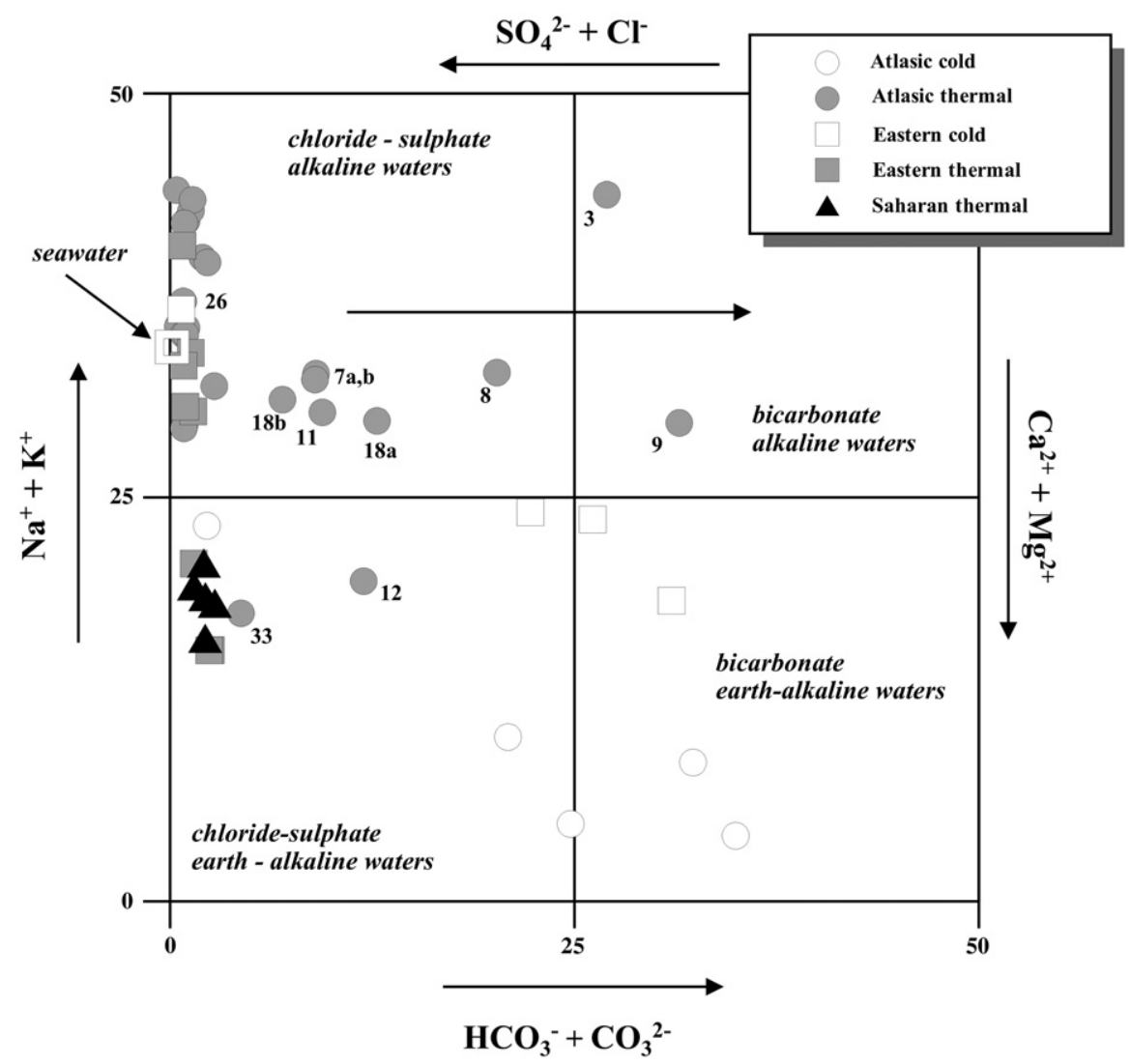

Fig. 2. Langelier-Ludwig classification diagram (Langelier and Ludwig, 1942) of Tunisian groundwaters. The numbers refer to the sampling sites. See text for explanations. 
$\mathbf{a}$

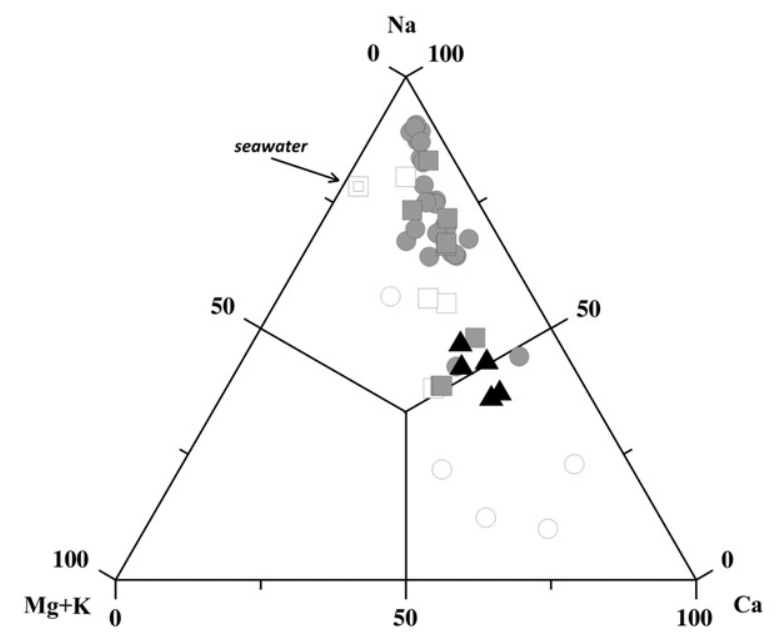

b

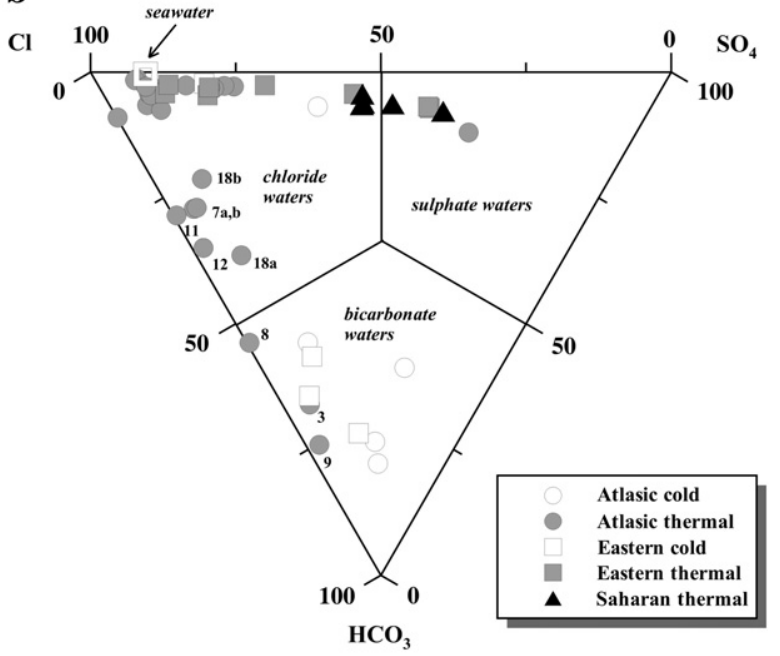

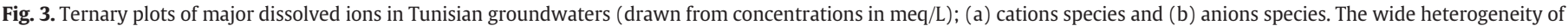
both cold and thermal waters composition is clearly supported by the two diagrams.

(iii) Thermal groundwaters from the Atlasic domain are dominantly Cl-type waters, but eventually span from $\mathrm{Cl}$-type to $\mathrm{HCO}_{3}$-type (Fig. 3b). The shift toward $\mathrm{HCO}_{3}$-type waters (Fig. 3b) reflects more a lower $\mathrm{Cl}$ content (relative to other samples of the Atlasic domain) than a real $\mathrm{HCO}_{3}$ increase (see Table 1 ), and is more manifest in a group of thermal groundwaters emerging in the Tellian sector (samples 3, 7a-b, 9, 11, 12, 18a-b);

(iv) Thermal groundwaters from the Eastern and Saharan domains are particularly $\mathrm{HCO}_{3}$-poor (relative to other anion species); in the triangular diagram of Fig. 3b, these samples define a fairly narrow (and almost continuous) compositional trend from $\mathrm{Cl}-$ type waters (Eastern domain) to $\mathrm{SO}_{4}$-type waters (Saharan Domain).

\subsection{Water isotopic composition}

The results of hydrogen and oxygen isotopic determinations in Tunisian groundwaters, expressed in $\delta$ units vs. V-SMOW, are listed in Table 1 . The $\delta \mathrm{D}$ and $\delta^{18} \mathrm{O}$ values are quite variable, ranging from -61.6 to $-15.6 \%$ and -8.48 to $-4.11 \%$, respectively. The $\delta \mathrm{D}-\delta^{18} \mathrm{O}$ scatter diagram (Fig. 4) confirms that all groundwaters are essentially meteoric in origin, with overall limited seawater contribution. The isotopic compositions of groundwaters from Northern (Atlasic + Eastern domains) and Southern (Saharan domain) Tunisia are in stark contrast (Fig. 4). The former have isotopic compositions falling within the field delimited by the Global Meteoric Water Line (GMWL; Craig, 1961 ) and the Eastern Mediterranean Meteoric Water Line (EMMWL; Gat and Carmi, 1970), and cluster in close proximity of the regional meteoric water lines derived by Celle-Jeanton et al. (2001) from local precipitations in Tunis and Sfax; the latter, on the contrary, plot to the right of the Global Meteoric Water Line and are characterized by $\delta D$ and $\delta^{18} \mathrm{O}$ values far more negative than the range of present-day precipitations. This depleted isotopic signature (with $\delta^{18} \mathrm{O}$ and $\delta \mathrm{D}$ values averaging at $-8.15 \%$ and $-59.2 \%$, respectively) is a distinctive feature of the Continental Intercalaire (CI) as shown by Guendouz et al. (1997), indicating that these groundwaters belong to a fossil deep reservoir whose recharge probably occurred early during the late Pleistocene period under different (colder and more humid) climatic conditions (Gonfiantini et al., 1974; Edmunds et al., 2003; Zouari et al., 2003; Trabelsi et al., 2009).

\subsection{Gas abundances}

Table 2 reports the relative abundances of the major gas species $\left(\mathrm{N}_{2}, \mathrm{O}_{2}, \mathrm{CO}_{2}, \mathrm{CO}, \mathrm{CH}_{4}, \mathrm{H}_{2}\right)$ in the dry gas phase at equilibrium with each water sample. The ternary plot of Fig. 5 shows that most Tunisian samples have $\mathrm{N}_{2}$-dominated compositions (from 33 to 99 vol.\%; Table 2), supporting a likely atmospheric derivation of the dissolved gasses. Oxygen represents only a relatively small fraction (0.06-15 vol.\%; Table 2); indeed, all samples are shifted towards the $\mathrm{N}_{2}$ corner relative to the representative compositional field of air. The relatively low $\mathrm{O}_{2}$ contents are not inconsistent with a main atmospheric derivation of the gasses, however : this oxygen depletion (relative to $\mathrm{N}_{2}$ ) is probably due to redox reactions between gasses and host rocks (Stumm and Morgan, 1996), and/or consumption of atmospheric oxygen by organic activity at shallow levels.

$\mathrm{CO}_{2}$ ranges from 0.2 to 40 vol.\%. Moderate $\mathrm{CO}_{2}$-excesses (Fig. 5), relative to atmospheric composition, are observed in several thermal waters from the Atlasic and Eastern domains; suggesting that these groundwater systems are open to contribution from an additional $\mathrm{CO}_{2}$ source than only atmosphere. The highest $\mathrm{CO}_{2}$ concentrations, from 20 to 40 vol.\% (e.g., samples 5a, 19 and 25 - see Table 2), correspond to $\mathrm{CO}_{2}$ partial pressures of $0.22,0.38$ and 0.51 atm, respectively, far exceeding those characteristic of the atmosphere $(0.00038 \mathrm{~atm})$ and soil (0.01 - 0.001 atm; Brook et al., 1983; Hamada and Tanaka, 2001). On the contrary, samples collected in the Saharan domain are systematically characterized by low $\mathrm{CO}_{2}$ contents (0.2-3 vol.\%).

\subsection{Carbon isotope geochemistry}

The total dissolved inorganic carbon contents of Tunisian groundwaters (TDIC, given by the sum of $\mathrm{CO}_{2 \mathrm{aq}}, \mathrm{HCO}_{3}$ and $\mathrm{CO}_{3}$ ) are listed in Table 2, together with their isotopic compositions $\left(\delta^{13} \mathrm{C}_{\text {TDIC }}\right)$. The TDIC values range from $2 \cdot 10^{-3}$ to $4 \cdot 10^{-2} \mathrm{~mol} \cdot \mathrm{L}^{-1}$, and $\delta^{13} C_{\text {TDIC }}$ from -11.23 and $+2.89 \%$ vs. V-PDB (Fig. 6a). This variability of compositions gives a hint for a range of different gaswater interaction environments, and supports a variety of different sources for dissolved carbon (Grassa et al., 2006).

The isotopic composition of TDIC represents an average of the isotopic compositions of the relevant dissolved carbon species, weighted by their respective contents in solution (Zhang et al., 1995). To derive insights into the origin of dissolved carbon in Tunisia 


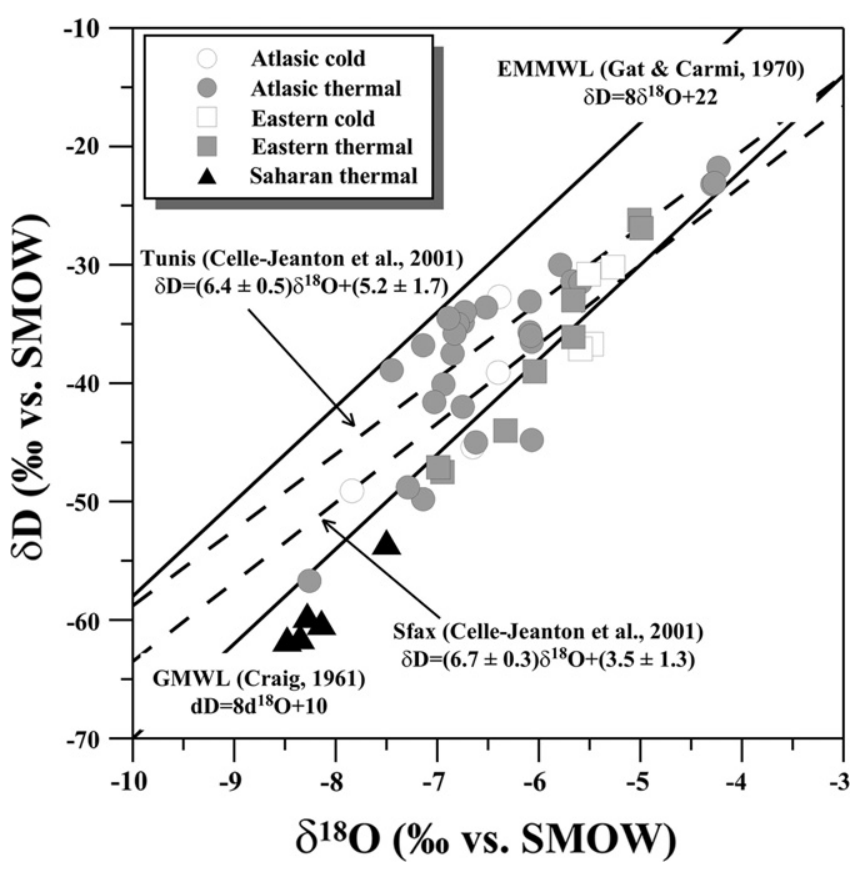

Fig. 4. $\delta \mathrm{D}$ vs. $\delta^{18} \mathrm{O}$ diagram of Tunisian groundwaters. The solid lines correspond to the Global Meteoric Water Line (GMWL; Craig, 1961) and the Eastern Mediterranean Meteoric Water Line (EMMWL; Gat and Carmi, 1970); while the dashed lines are indicative of $\delta \mathrm{D}$ and $\delta^{18} \mathrm{O}$ isotopic compositions of local rainwaters in Tunis and Sfax (Celle-Jeanton et al., 2001).

groundwaters, the isotopic compositions of gaseous $\mathrm{CO}_{2}$ at isotopic equilibrium with each water sample (at the given T, pH and TDIC) have been calculated by Eq. (1). This relation takes into account the dependence of equilibrium fractionations between gaseous $\mathrm{CO}_{2}$ and the different dissolved carbon species $\left(\mathrm{CO}_{2(a q)}, \mathrm{HCO}_{3(a q)}^{-}\right.$and $\left.\mathrm{CO}_{3(a q)}^{2-}\right)$. The extent of these TDIC- $\mathrm{CO}_{2}(\mathrm{~g})$ fractionations thus depends on $\mathrm{T}$ and

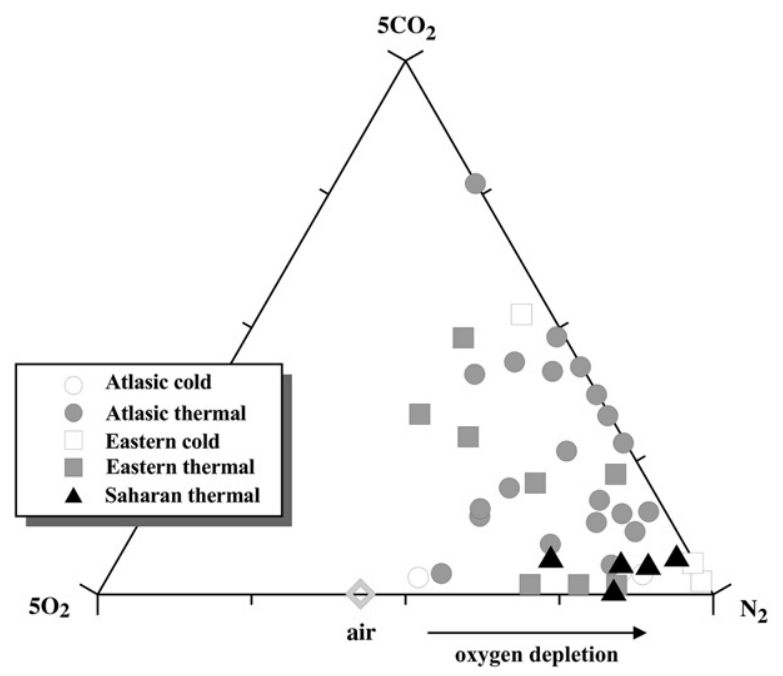

Fig. 5. Ternary plot of the main species $\left(\mathrm{N}_{2}, \mathrm{CO}_{2}\right.$ and $\left.\mathrm{O}_{2}\right)$ in gas phase at equilibrium with each groundwater sample, drawn from the volumetric percentage (vol.\%) reported in Table 2. Tunisian groundwaters display $\mathrm{N}_{2}$-dominated compositions, and are characterized by oxygen depletion relative to air composition (likely due to redox reactions in the hydro-cycle). They also manifest $\mathrm{CO}_{2}$-enrichment (relative to air), pointing to the existence of additional $\mathrm{CO}_{2}$ sources.
$\mathrm{pH}$ of the solutions, and from the enrichment factor $(\varepsilon)$ of each species with respect to gaseous $\mathrm{CO}_{2}$ (Zhang et al., 1995).

$$
\begin{aligned}
\delta^{13} \mathrm{C}_{\mathrm{CO} 2 \mathrm{~g}}= & \delta^{13} \mathrm{C}_{\mathrm{TDIC}}-\left(\varepsilon_{\mathrm{CO} 2 \mathrm{aq}-\mathrm{CO} 2 \mathrm{~g}} \times \chi_{\mathrm{CO} 2 \mathrm{aq}}\right)-\left(\varepsilon_{\mathrm{HCO}-\mathrm{CO} 2 \mathrm{~g}} \times \chi_{\mathrm{HCO} 3}\right) \\
& -\left(\varepsilon_{\mathrm{CO} 3-\mathrm{CO} 2 \mathrm{~g}} \times \chi_{\mathrm{CO} 3}\right)
\end{aligned}
$$

where the different enrichment factor values $(\varepsilon)$ are taken from Deines et al. (1974) and $\chi_{\mathrm{CO} 2 \mathrm{aq}}, \chi_{\mathrm{H} 2 \mathrm{CO} 3}$ and $\chi_{\mathrm{CO} 3}$ are the equilibrium molar ratios of aqueous carbon species at the same conditions.

The so-obtained $\delta^{13} \mathrm{C}_{\mathrm{CO} 2}$ values (Table 2) range from -17.15 to $-0.93 \%$ vs. V-PDB, and are plotted in Fig. $6 \mathrm{~b}$ against the corresponding $\mathrm{CO}_{2}$ content. Fig. $6 \mathrm{~b}$ shows that $\mathrm{CO}_{2}$ isotopic compositions become more positive upon increasing $\mathrm{CO}_{2}$ content. Samples from the Saharan domain, being the most $\mathrm{CO}_{2}$-poor (see also Fig. 5), are also characterized by the most ${ }^{13} \mathrm{C}$-depleted isotopic compositions $\left(\delta^{13} \mathrm{C}_{\mathrm{CO} 2}<-9 \%\right.$ vs. V-PDB), and approach the typical compositions of organically-derived $\mathrm{CO}_{2}\left(\delta^{13} \mathrm{C}_{\mathrm{CO} 2 \text {, bio }}<-18 \%\right.$ vs. V-PDB $)$. We stress, however, the lack of very negative $(<-18 \%$ vs. V-PDB) samples in our dataset, which we ascribe to both the "buffering" action played by calcite dissolution (see Section 5.2), and to the fact that soil cover is relatively poorly developed in arid regions like Tunisia (thus reducing biologic $\mathrm{CO}_{2}$ production in the soil environment). Intermediate $\mathrm{C}$ isotopic compositions are observed for a large cluster of samples which, in spite of their relatively minor $\mathrm{CO}_{2}$ content $\left(\chi_{\mathrm{CO} 2}<0.1 \mathrm{~mol} \cdot \mathrm{mol}^{-1}\right)$, spread in $\delta^{13} \mathrm{C}_{\mathrm{CO} 2}$ from -10 to $-5 \%$ vs. V-PDB. Finally, the less negative isotopic compositions $\left(\delta^{13} \mathrm{C}_{\mathrm{CO} 2}\right.$ of -4 to $-1 \%$ vs. V-PDB) correspond to the most $\mathrm{CO}_{2}$-rich samples from the Atlasic and Eastern domains.

Fig. $6 \mathrm{~b}$ compares our derived $\mathrm{CO}_{2}$ isotopic compositions of Tunisian groundwaters with analogous data from volcanic (Pantelleria Island) and non-volcanic districts of nearby Sicily. This shows that Tunisian groundwaters have $\delta^{13} \mathrm{C}_{\mathrm{CO} 2}$ compositions overlapping the measured compositional range of thermal waters from central-western Sicily, an area of remarkably similar geodynamic context (Grassa et al., 2006); while they are strongly dissimilar in composition (more ${ }^{13} \mathrm{C}$-rich) to $\mathrm{CO}_{2}$ in thermal waters from Pantelleria (Parello et al., 2000), in the Sicily channel rift (Fig. 1).

\subsection{Helium isotopes}

Helium isotopes results are given in Table 2. All $\left({ }^{3} \mathrm{He} /{ }^{4} \mathrm{He}\right)_{\mathrm{xs}}$ values, where $\left({ }^{3} \mathrm{He} /{ }^{4} \mathrm{He}\right)_{\mathrm{xs}}$ is the isotopic ratio of non-atmospheric helium deduced from the neon correction, are below the atmospheric ratio (Ra) except for sample 25 (Ain Garci site) which reaches $2.4 \mathrm{Ra}$ (Fig. 7). When gas and water were sampled simultaneously, we observe a good agreement between both isotopic ratios (Table 3).

The lowest ${ }^{3} \mathrm{He} /{ }^{4} \mathrm{He}$ values, comparable to the crustal production ratio of 0.01-0.05 Ra (Ballentine and Burnard, 2002), are found in the central and southern parts of the country (Figs. 7 and 8), associated with the Atlasic domain and the Saharan Platform, respectively. In the Tellian sector (northwestern Tunisia), the ${ }^{3} \mathrm{He} /{ }^{4} \mathrm{He}$ values are still quite low $(0.13 \pm 0.08 \mathrm{Ra})$ but clearly above the crustal ratio. West of the Tellian sector, the Marwa site (sample 14) and the Ichkeul group (samples 15-16-17) display higher ${ }^{3} \mathrm{He} /{ }^{4} \mathrm{He}$ values, around $0.4 \mathrm{Ra}$ (Figs. 7 and 8). In the Eastern Domain, ${ }^{3} \mathrm{He} /{ }^{4} \mathrm{He}$ values range from 0.25 to $0.65 \mathrm{Ra}$ and up to $2.4 \mathrm{Ra}$ in Ain Garci (sample 25), a $\mathrm{CO}_{2}$ rich mineral spring located some $30 \mathrm{~km}$ south of the city of Zaghouan. For this water, the low tritium (1.2 $\pm 0.1 \mathrm{TU}$ - Fourré et al., in prep.) and high helium $\left(135 \times 10^{-8} \mathrm{~cm}^{3} \mathrm{STP} / \mathrm{g}\right)$ contents rule out any significant tritiogenic ${ }^{3} \mathrm{He}$ contribution to the elevated ${ }^{3} \mathrm{He} /{ }^{4} \mathrm{He}$ ratio.

Measured helium concentrations are well above the air-saturated value (4-5 $\times 10^{-8} \mathrm{~cm}^{3} \mathrm{STP} / \mathrm{g}$ ), ranging from 7.6 to $4723 \times 10^{-8} \mathrm{~cm}^{3} \mathrm{STP} / \mathrm{g}$ (Table 2), except for sample 5 (Hammam Biadha) where values measured in 2006 and again in 2009 are below the atmospheric solubility 

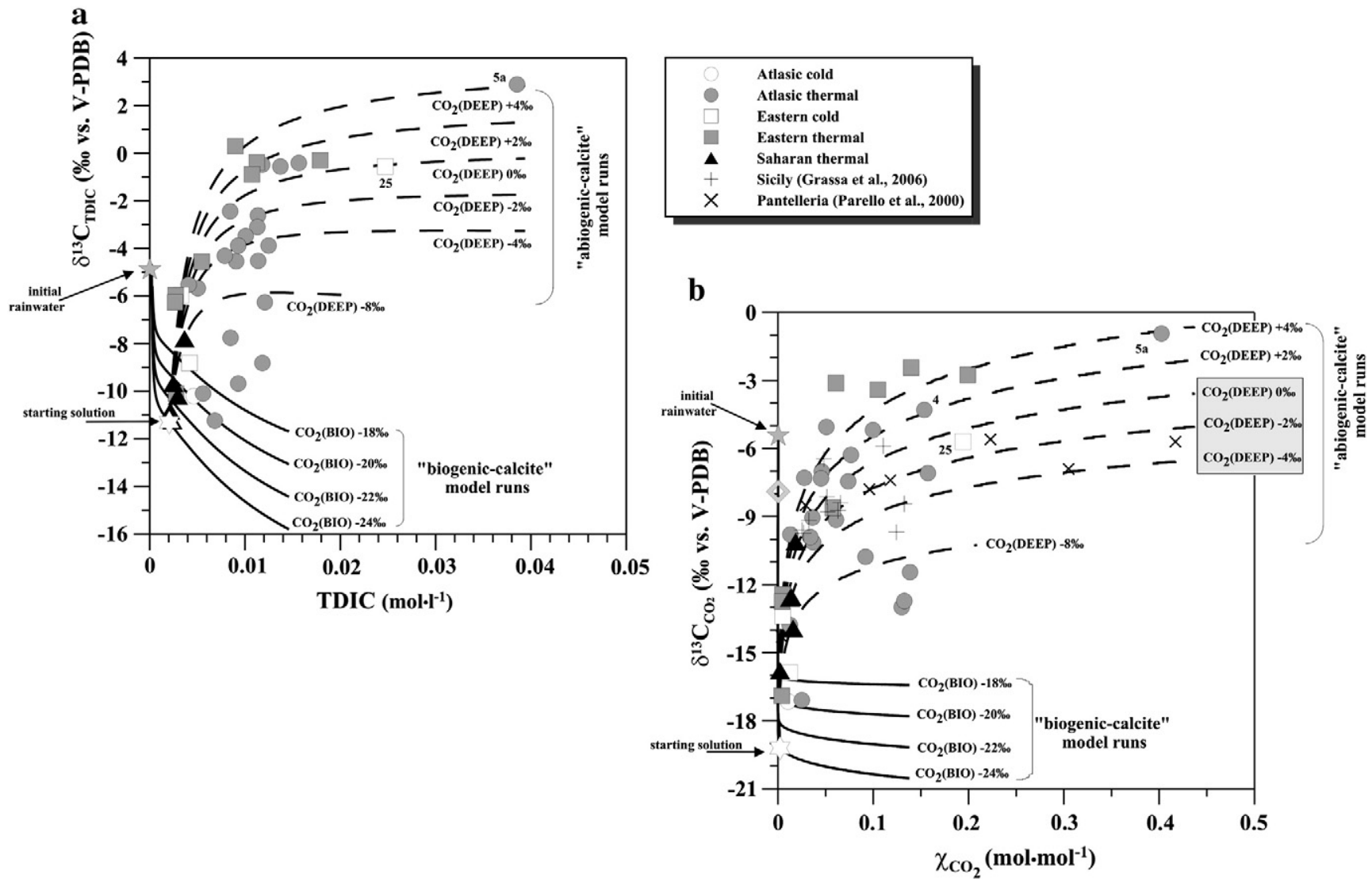

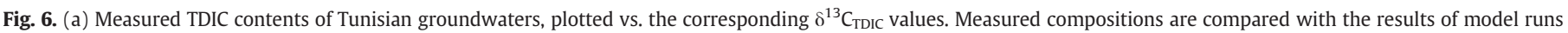

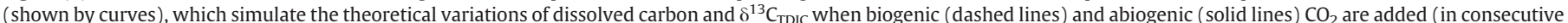

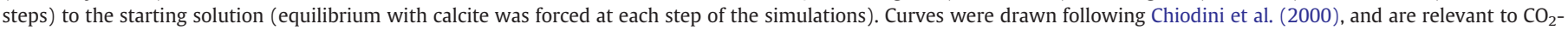

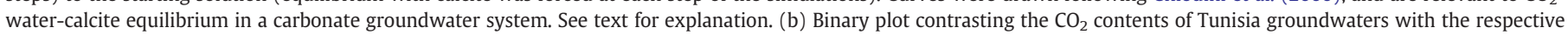

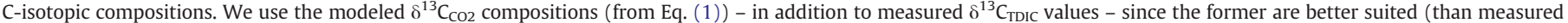

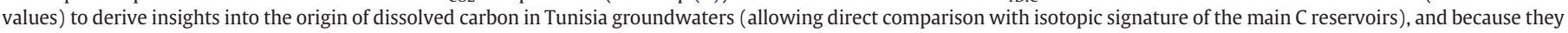

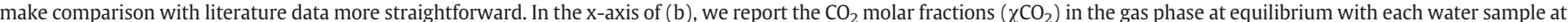

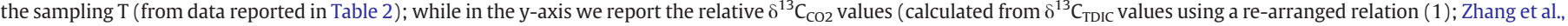

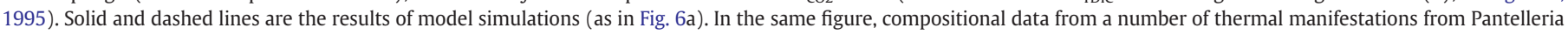

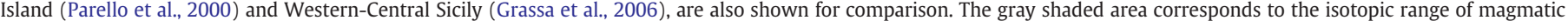
carbon in Italy.

value, probably due to helium stripping by the strong $\mathrm{CO}_{2}$ bubbling which occurs at this site. Particularly high values, in the range 1777$4723 \times 10^{-8} \mathrm{~cm}^{3} \mathrm{STP} / \mathrm{g}$ (samples $26,29,30,34,35$ ) are observed on a SW-NE transect across central Tunisia going from Gafsa to Sousse (Fig. 8), associated with well documented tectonic features (see discussion below).

\section{6. $\mathrm{He}-\mathrm{CO}_{2}$ characteristics}

Fig. 9 displays the $\mathrm{CO}_{2}$-helium results plotted on a $\mathrm{CO}_{2}-{ }^{4} \mathrm{He}-{ }^{3} \mathrm{He}$ diagram (after Giggenbach et al., 1993). The $\mathrm{CO}_{2} /{ }^{3} \mathrm{He}$ ratios range from $2.6 \times 10^{6}$ to $3.5 \times 10^{11}$. In contrast with typical volcanic environments where helium and $\mathrm{CO}_{2}$ relationship results in rather well defined $\mathrm{CO}_{2} /{ }^{3} \mathrm{He}$ values (Hilton et al., 2002), the Tunisian data set shows five orders of magnitude variability, going from lower than MORB values $\left(\mathrm{CO}_{2} /{ }^{3} \mathrm{He}=2 \times 10^{9}\right)$ to crustal values $\left(\mathrm{CO}_{2} /{ }^{3} \mathrm{He}>10^{10}\right)$, with most values however characterized by $\mathrm{CO}_{2} /{ }^{3} \mathrm{He}$ ratios between the typical MORB $\left(\mathrm{CO}_{2} /{ }^{3} \mathrm{He}=2 \times 10^{9}\right)$ and global average arc values $\left(\mathrm{CO}_{2} /{ }^{3} \mathrm{He}=1.2 \times 10^{10}\right)$. This wide variability is best explained, as discussed below (see Section 5.2 ), by the fact that the respective origin of $\mathrm{CO}_{2}$ and helium isotopes are largely decoupled in the system, with most samples characterized by crustal helium and metamorphic $\mathrm{CO}_{2}$ mixed in variable amounts.

\section{Discussion}

\subsection{Geochemical processes : sources of salts and link with geology}

From a chemical viewpoint, the most striking feature of Tunisian thermal waters is their large heterogeneity (see Figs. 2 and 3). This is indeed not surprising in light of the variability of geological landforms, terrains, and structural settings in the various parts of the country. In most geothermal fluids hosted by active volcanic systems (Goff and Janik, 2000), the sources of dissolved chemicals include (i) the interaction of deep-rising magmatic gasses (sourcing the majority of $\mathrm{Cl}, \mathrm{S}$ and $\mathrm{C}$ ) with infiltrating surface waters (formerly meteoric or marine in origin), and (ii) the resultant high-temperature gas-water-silicate hydrolysis reactions, which supply cation species to hydrothermal solutions. In these hydrothermal systems, the contrasting chemical compositions of the various surface manifestations generally arise from the fact that they correspond to different steps in the maturation path of infiltrating waters (Giggenbach, 1988). In sedimentary environments such as Tunisia, however, the sources of chemicals in thermal waters are more challenging to interpret, because the mineralogical and chemical properties of the hosting aquifers are inherently more heterogeneous, and because physical (e.g., heat flux, stress regime) and hydrological conditions (e.g., infiltration depth and residence time in reservoir) are likely to be 


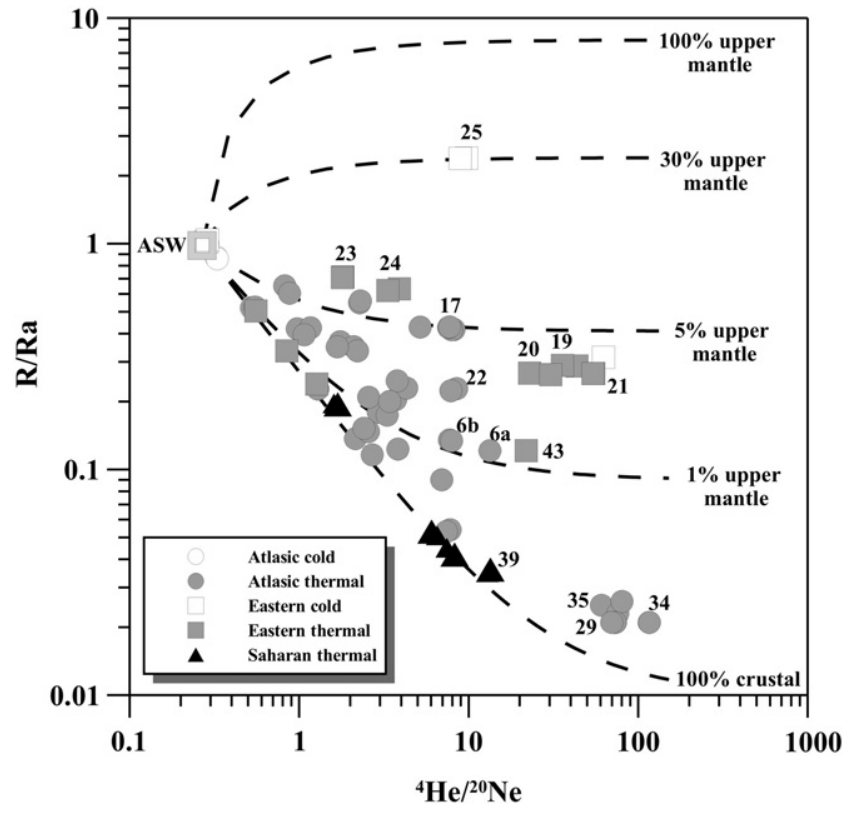

Fig. 7. Helium isotopes results plotted as R/Ra vs. ${ }^{4} \mathrm{He} /{ }^{20} \mathrm{Ne}$. The dotted lines depict mixing trends between the atmospheric and the crustal components, with various addition of an upper mantle component with $\mathrm{R}=8 \times \mathrm{Ra}$.

different and specific to each manifestation. In view of this complexity, and taking into account that our regional-scale geochemical prospecting precludes detailed investigation of each single manifestation, we attempt below at discussing the key control factors/processes in the evolution of Tunisian thermal waters.

First, we observe that most thermal waters from the Atlasic and Eastern domains have a prevalent $\mathrm{NaCl}$ composition (Figs. 2 and 3). Accepting the fact that groundwater-seawater interactions are modest (Fig. 4) if not locally near the coast, and that contribution from high-T Cl-bearing magmatic gasses is negligible (as indicated by the low ${ }^{3} \mathrm{He} /{ }^{4} \mathrm{He}$ ratios), we argue then that the interaction of infiltrating waters with halite-bearing Triassic evaporites is the most likely source of both $\mathrm{Na}$ and $\mathrm{Cl}$. There is a fair positive correlation between sodium and chlorine contents (Fig. 10a), and Cl-rich waters $\left(\mathrm{Cl} / \sum\right.$ anions $>0.7$; see Table 1$)$ belonging to the Atlasic and Eastern domains are all characterized by molar $\mathrm{Na} / \mathrm{Cl}$ ratios of $\sim 1$ (the stochiometric $\mathrm{Na} / \mathrm{Cl}$ ratio of halite dissolution; Fig. 10a). These groundwaters have Saturation Indexes $(\mathrm{SI})$ for halite $\left(\mathrm{SI}_{\text {Halite }}\right)$ from -6 to -2 (Table 1 ), suggesting they have extensively leached soluble salts, though not having reached equilibrium with halite, yet.

Secondly, we identify a typical $\mathrm{SO}_{4}$-type composition for the thermal waters from the Saharan domain. Most of these waters were collected from deep artesian wells tapping the $\mathrm{CI}$, and thus consistently have the typical $\mathrm{Na}, \mathrm{Ca}-\mathrm{SO}_{4}$ composition (see Fig. 3), as already revealed by earlier studies (Edmunds et al., 2003). Clearly, this composition highlights that dissolution of gypsum and/or anhydrite, which are minor but recurring constituents of the $\mathrm{CI}$ sandstone formations, plays a major role in groundwater mineralization. It is not surprising to observe then, that all thermal waters from the Saharan domain cluster along the gypsum/anhydrite dissolution line (at equivalent $\mathrm{Ca}$ to $\mathrm{SO}_{4}$ ratios of 1:1: see Fig. $10 \mathrm{~b}$ ), and are close to equilibrium with gypsum ( $\mathrm{SI}_{\text {gypsum }}$ of -0.6 to -0.4 ; Table 1 ). The same Fig. 10b reveals that several thermal waters from the Atlasic and Eastern domains also plot along the same gypsum/anhydrite dissolution line. This suggests that dissolution of sulfate minerals, likely hosted in the Triassic evaporitic sequence and/or in the upper Cretaceous series (Fig. 1), contributes to water chemistry also in these parts of the country.

Thirdly, carbonate formations are widespread all over Tunisia (included in the Triassic evaporitic sequence and in the Cretaceous to Cenozoic limestones). Yet, carbonate mineral dissolution visibly affects water chemistry only where more-easily leachable evaporitic minerals are absent (or limited). It is noteworthy to observe, in fact, that while a relatively narrow band of Triassic evaporites forms the NE-SW-trending "diapir zone" extending from Bizerte to Le Kef (see black-colored band in Fig. 1), no major Triassic outcrop is reported in the Tellian sector (northernmost part of the Atlasic domain; Fig. 1), where Oligo-Miocene Numidian Flysch units mainly crop out : thermal waters collected in this part of the country (samples 3, 7a-b, 9, 11, 12, 18a-b; see Fig. 1) consistently depart from the gypsum/anhydrite dissolution line (Fig. 10b), implying that evaporitic mineral dissolution is an unquestionably more marginal process : they have dominant $\mathrm{Na}$, $\mathrm{Ca}-\mathrm{HCO}_{3}$ compositions (Fig. 3), and display $\mathrm{Ca} / \mathrm{HCO}_{3}$ ratios of $\sim 1$ (see cluster of data points falling within the white area in Fig. 10c), typical of waters undergoing calcite dissolution as the prevalent mineralization process. These water samples consistently are at equilibrium conditions with calcite $(-0.4<\mathrm{SI}<0.6)$.

The key source processes controlling the chemistry of Tunisian thermal groundwaters are summarized in Fig. 10d. This confirms that $\mathrm{Na}$ and $\mathrm{Cl}$ concentrations are primarily controlled by dissolution of the saliferous Triassic formations rich in halite (Iundt, 1971; Edmunds et al., 2003; Kamel et al., 2005; Guendouz and Michelot, 2006; Inoubli et al., 2006; Trabelsi et al., 2009), which buffers the $\mathrm{Na} / \mathrm{Cl}$ ratio of most samples at $\sim 1$. This process is active over the three Tunisian domains. In addition to this, leaching of gypsum/anhydrite and calcite concurs to determine the range of $\mathrm{HCO}_{3} /\left(\mathrm{Ca}+\mathrm{SO}_{4}\right)$ ratios in groundwaters: sulfate dissolution, which determines a shift of groundwater samples towards the left part of Fig. 10d, appears dominant in Saharan and Eastern domains $\left(\mathrm{HCO}_{3} /\left(\mathrm{Ca}+\mathrm{SO}_{4}\right)\right.$ ratios $\left.<0.1\right)$; while calcite dissolution (which acts to produce a shift towards the right portion of Fig. 10d), mainly controls water chemistry in the Atlasic Domain

Table 3

Helium isotopes composition of bubbling free gas samples compared to dissolved gas in corresponding groundwaters.

\begin{tabular}{|c|c|c|c|c|c|c|c|c|c|}
\hline \multirow[t]{3}{*}{ Sample } & \multirow[t]{3}{*}{ Site name } & \multicolumn{2}{|l|}{ Water } & \multicolumn{3}{|l|}{ Gas } & \multicolumn{2}{|l|}{ Water } & \multirow{3}{*}{$\frac{\text { Gas }}{\mathrm{R}_{\mathrm{xs}} / \mathrm{Ra}}$} \\
\hline & & ${ }^{4} \mathrm{He}_{\mathrm{xs}}(\mathrm{ppm})$ & $\overline{{ }^{4} \mathrm{He}_{\mathrm{xs}}(\mathrm{ppm})}$ & ${ }^{4} \mathrm{He}(\mathrm{ppm})$ & ${ }^{4} \mathrm{He} /{ }^{20} \mathrm{Ne}_{\text {meas }}$ & $\overline{{ }^{4} \mathrm{He}_{\mathrm{xs}}(\mathrm{ppm})}$ & $\mathrm{R}_{\mathrm{xs}} / \mathrm{Ra}$ & $\overline{\mathrm{R}_{\mathrm{xS}} / \mathrm{Ra}}$ & \\
\hline & & (1) & $(2)$ & & & & $(1)$ & $(2)$ & \\
\hline $5 a$ & H. Biadha & 3.2 & 4.0 & 7.8 & 38.44 & 7.7 & 0.262 & 0.191 & 0.178 \\
\hline $5 b$ & H. Biadha & 3.6 & 3.3 & 4.9 & 26.08 & 4.8 & 0.179 & 0.231 & 0.185 \\
\hline $7 a$ & H. Es-Salhine & 19.0 & & 32.6 & 3.43 & 29.6 & 0.260 & & 0.245 \\
\hline $7 b$ & H. Es-Salhine & 22.2 & 23.5 & 39.6 & 3.75 & 36.2 & 0.259 & 0.248 & 0.129 \\
\hline $13 a$ & H. Kef-Ettout & 50.3 & 91.7 & 46.1 & 4.01 & 42.4 & 0.023 & 0.060 & 0.017 \\
\hline $13 b$ & H. Kef-Ettout & 17.4 & & 40.1 & 4.12 & 37.0 & 0.033 & & 0.028 \\
\hline 29 & A. Maamar (H. Sollah) & 3166 & 3736 & 3236 & 134.76 & 3228 & 0.018 & 0.018 & 0.022 \\
\hline 30 & A. Chenama (A. Mouailha) & 4141 & 5161 & 3023 & 135.72 & 3016 & 0.021 & 0.023 & 0.028 \\
\hline
\end{tabular}

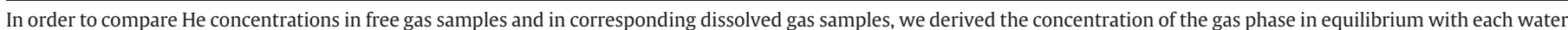

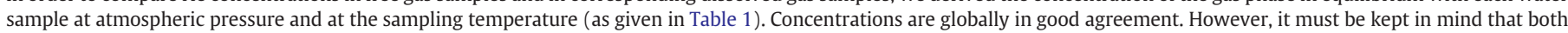
water degassing in the hydrothermal system before sampling and/or gas high-pressure in the hydrothermal system inducing higher solubility can play in opposite ways. 

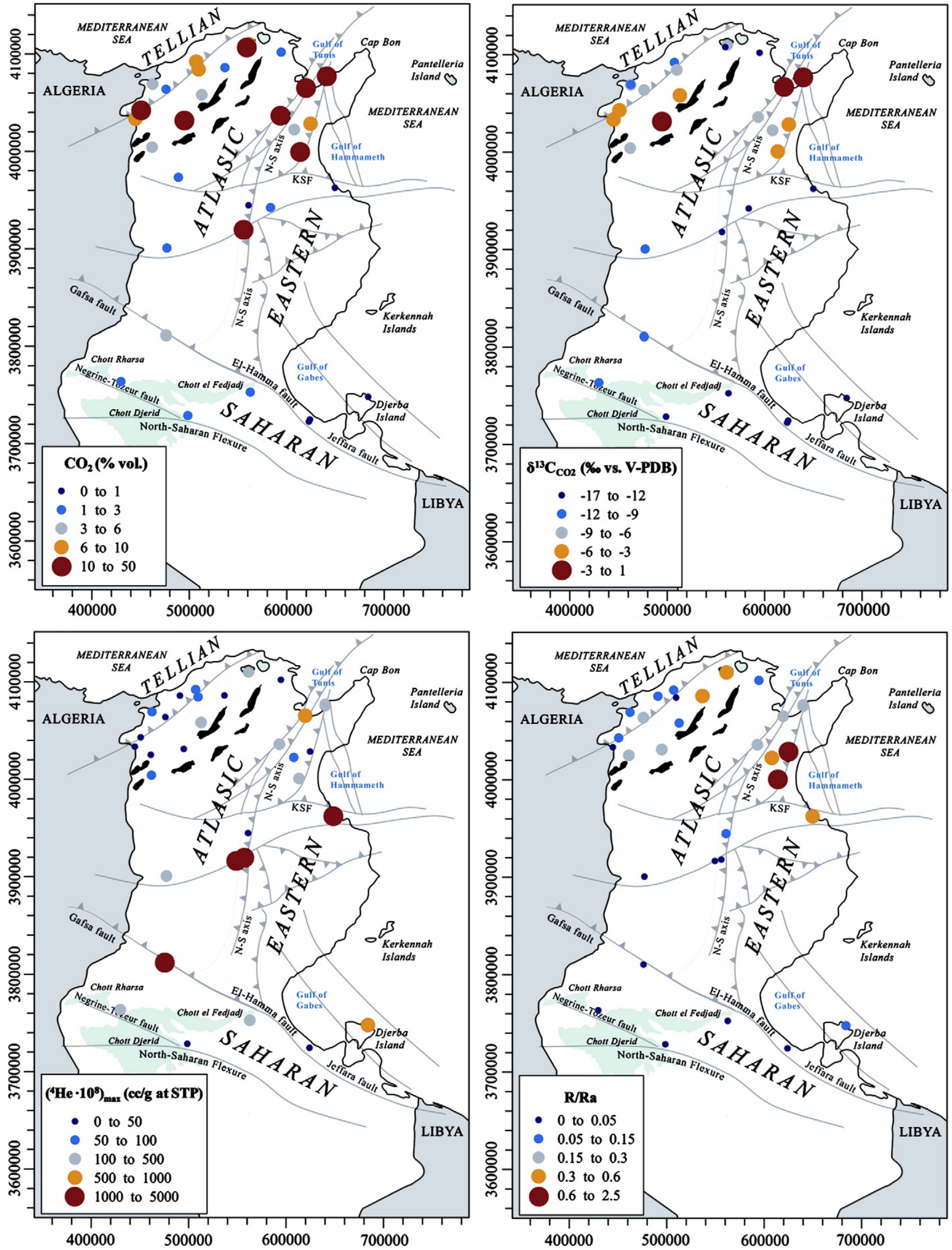

Fig. 8. Maps showing the spatial distribution of $\mathrm{CO}_{2}, \delta^{13} \mathrm{C},{ }^{4} \mathrm{He}$ and $\mathrm{R} / \mathrm{Ra}$ in Tunisia and their connection to tectonics. 


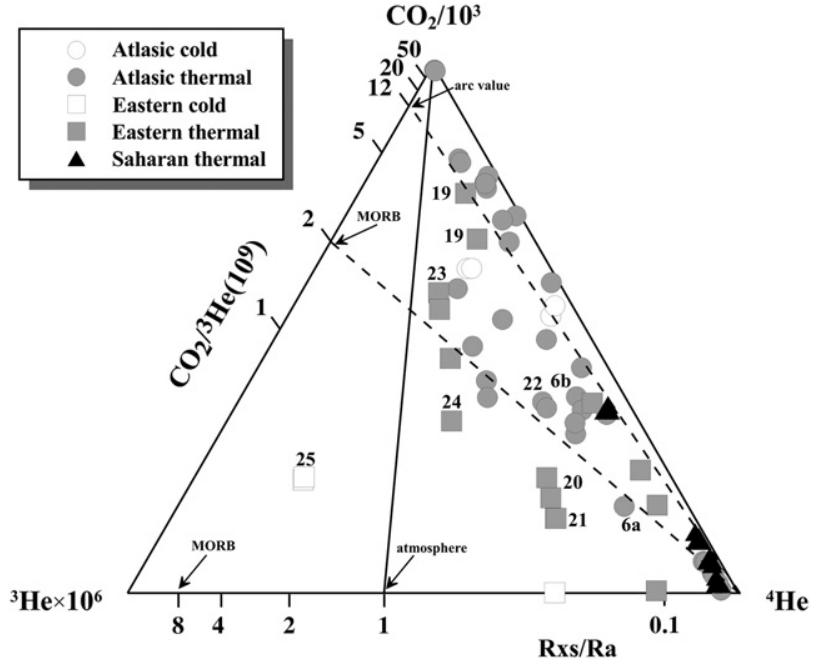

Fig. 9. Ternary plot of $\mathrm{CO}_{2}-{ }^{3} \mathrm{He}-{ }^{4} \mathrm{He}$. Solid line represents a mixture of atmospheric $\mathrm{He}$ (1Ra) and pure $\mathrm{CO}_{2}$. Dashed lines represent mixture between radiogenic $\mathrm{He}$ and $\mathrm{CO}_{2}{ }^{3} \mathrm{He}$ with average arc ratio $\left(1.2 \times 10^{10}\right.$; Sano and Williams, 1996) and average MORB ratio $\left(2 \times 10^{9}\right.$; Marty and Jambon, 1987$)$ respectively.

$\left(\mathrm{HCO}_{3} /\left(\mathrm{Ca}+\mathrm{SO}_{4}\right)\right.$ ratios $\left.>0.1\right)$, and more specifically in its Tellian sector (where $\mathrm{HCO}_{3} /\left(\mathrm{Ca}+\mathrm{SO}_{4}\right)$ ratios occasionally are $>1$ ). In the latter area, the absence of halite-bearing formations in the sedimentary succession allows the groundwater $\mathrm{Na} / \mathrm{Cl}$ ratio to significantly diverge from unity. This Na-excess (relative to $\mathrm{Cl}$ ) (see samples 3 , 8 and 9 in Fig. 10d) likely reflects weathering of feldspars (mainly from sandstones of the Numidian Flysch; Garcia et al., 2001). We cannot exclude, however, that cation exchange processes on mineral surfaces also play a role : these are known to cause a preferential $\mathrm{Na}$ release to weathering solutions at the expense of other cation species (Edmunds et al., 2003; Guendouz et al., 2003; Yermani et al., 2003; Fehdi et al., 2009; Kouzana et al., 2010).

\subsection{Origin of carbon and helium}

The isotopic compositions of dissolved carbon and helium are known to be sensitive tracers of the origin of volatiles in subsurface fluids. In the following, we examine the possible origin for carbon in the light of the $\delta^{13} \mathrm{C}$ composition of the fluids, and the spatial distribution of He isotopes in relation to the structural geology of Tunisia.

\subsubsection{Carbon isotopes}

The determination of $\delta^{13} C_{\text {TDIC }}$ was undertaken to gain insights into the processes controlling the abundance and origin of dissolved carbon in the thermal waters. As shown in Section 4.4, the highly variable carbon isotopic signature of TDIC (Fig. 6a) and $\mathrm{CO}_{2}$ content (Fig. 6b) point to multiple carbon sources. In the attempt to evaluate the relative contributions from these sources, we follow here the modeling approach developed by Chiodini et al. (2000) to numerically simulate the evolution of the TDIC and $\delta^{13} C_{\mathrm{TDIC}}$ of infiltrating meteoric waters in the $\mathrm{CO}_{2(\mathrm{~g})}$-calcite-water system. The Chiodini et al. (2000) model, developed to investigate the origin of carbon dissolved in groundwaters from the Apennines (Central Italy), stems from the hypothesis that, in tectonically-active sedimentary groundwater systems, the most significant sources of carbon may include : (i) carbonate minerals (all our samples are at equilibrium with calcite as shown by the SI calcite values in Table 1), (ii) organically-derived $\mathrm{CO}_{2}$ (taken up upon interaction with the organic pool of the soil, or possibly coming from organic carbon buried in deeper geological layers) and, (iii) possibly, deeprising abiogenic $\mathrm{CO}_{2}$. The model was numerically solved using the
PHREEQC software package (Parkhurst and Appelo, 1999), and the results are reported in Fig. 6 .

Two sets of simulations were carried out. In a first set of "biogeniccalcite" model runs, we used as the starting solution an hypothetic rainwater sample having a $\mathrm{TDIC}_{\text {rain }}$ of $1.48 \cdot 10^{-5} \mathrm{~mol} \cdot \mathrm{L}^{-1}$ (corresponding to the equilibrium TDIC content in the water-atmospheric $\mathrm{CO}_{2}$ system at $20{ }^{\circ} \mathrm{C}$ and $\mathrm{pH}=5.6$ ), and a $\delta^{13} \mathrm{C}_{\mathrm{TDIC}, \text { rain }}$ of $-4.88 \%$ 。 (corresponding to the calculated $\mathrm{C}$-isotopic composition of pure water in equilibrium with atmospheric $\mathrm{CO}_{2(\mathrm{~g})}$, this having a $\delta^{13} \mathrm{C}$ of $-7 \%$ ) (see Fig. 6a). We then assumed that, upon infiltration, the starting rainwater solution interacts with organically-derived $\mathrm{CO}_{2}\left(\mathrm{CO}_{2, \text { bio }}\right)$ : this process was simulated by addition, in 200 steps, of $0.01 \mathrm{~mol}$ of $\mathrm{CO}_{2 \text {,bio }}$ (to $1 \mathrm{~kg}$ of water), which isotopic composition $\left(\delta^{13} \mathrm{CO}_{2 \text {,bio }}\right)$ was varied from $-18 \%$ to $-24 \%$ o (Chiodini et al., 2000). At each step, equilibrium with calcite $\left(\delta^{13} C_{\text {carb }}=+2 \%\right.$; Chiodini et al., 2000) was imposed (calcite was only allowed to dissolve in - and not to precipitate from - the solution). In the assumption that (i) isotopic equilibrium exists between all of dissolved carbon species, (ii) $\mathrm{CO}_{2}$ is not degassed from solution (no $\mathrm{CO}_{2}$ output), and (iii) no isotopic fractionation occurs when $\mathrm{CO}_{2}$ is added to the initial solution, the TDIC and $\delta^{13} \mathrm{C}_{\mathrm{TDIC}}$ of model solutions can be calculated using the relations proposed by Wigley et al. (1978). The results of the "biogenic-calcite" model runs are reproduced by the " $\mathrm{CO}_{2}$ (BIO)" solid lines in Figs. 6a,b. These show that, when biogenic $\mathrm{CO}_{2}$ is added to a calcite-buffered water system, the TDIC contents of model solutions increase at each step, while the respective $\delta^{13} \mathrm{C}_{\mathrm{TDIC}}$ (Fig. 6a) consistently become more negative. The chemical $\left(\chi_{\mathrm{CO} 2}\right)$ and isotopic $\left(\delta^{13} \mathrm{C}_{\mathrm{CO} 2}\right)$ composition of the gas phase at equilibrium with model solutions, shown in Fig. 6b, evolve following parallel trends towards more ${ }^{13} \mathrm{C}$-poor compositions. Comparison between modeled trends and measured compositions (Fig. 6a,b) indicates, however, that only a relatively limited number of Tunisian groundwaters (mostly from the $\mathrm{CI}$ ) can be satisfactorily reproduced by this process; while most of the remaining samples have far more positive $\delta{ }^{13} \mathrm{C}_{\mathrm{TDIC}}$ and $\delta^{13} \mathrm{C}_{\mathrm{CO} 2}$ values, implying than an additional ${ }^{13} \mathrm{C}$-rich carbon source must be at play.

A second set of "abiogenic-calcite" model runs was therefore carried out, in which a ${ }^{13} \mathrm{C}$-rich $\mathrm{CO}_{2}$ (with $\delta^{13} \mathrm{C}$ values ranging from -8 to $+4 \%$ ) was stepwise added to the starting solution (instead of the ${ }^{13} \mathrm{C}$-poor biogenic $\mathrm{CO}_{2}$ ). As a starting solution for these simulations, we used a moderately $\mathrm{C}$-rich water (indicated by the star in Fig. 6) from one of the "biogenic-calcite" runs: this allows us to account for a small contribution from soil biogenic $\mathrm{CO}_{2}$ in the first steps of the water infiltration process (sensitivity tests show that the model results are almost unaffected by the choice of the starting solution which contributes indeed to a very low fraction of the total modeled carbon). Again, calcite equilibration was forced at each step of the simulation. Note that the effect of $\mathrm{CO}_{2}$ degassing (Chiodini et al., 2000; Caliro et al., 2005) on the model runs is not considered here, since this process has negligible effects on the $\mathrm{C}$ isotopic balance at the low carbon conditions studied here (stepwise addition of a cumulative $\mathrm{CO}_{2}$ amount of only $0.03 \mathrm{~mol}$ ). The model solutions derived from the "abiogenic-calcite" runs, graphically illustrated by the family of dashed lines in Figs. $6 a$ and $b$, have compositions overlapping the measured TDIC- $\delta{ }^{13} C_{\mathrm{TDIC}}\left(\chi_{\mathrm{CO} 2}-\delta{ }^{13} \mathrm{C}_{\mathrm{CO} 2}\right)$ range for most water samples from the Atlasic and Eastern domains.

Overall, comparison between modeled and natural water compositions clearly highlights that some abiogenic carbon reservoir must be sourcing a relevant fraction of the carbon transported by the Tunisian groundwaters. In order to fit the measured composition of the $\mathrm{CO}_{2}$-richest Tunisian thermal waters (Fig. 6), this abiogenic $\mathrm{CO}_{2}$ must be isotopically positive, and most-probably characterized by $\delta^{13} \mathrm{C}_{\mathrm{CO} 2}$ values between 0 and $+4 \%$ 。 vs. V-PDB. This is far beyond the accepted isotopic range of magmatic $\mathrm{CO}_{2}$, which is comprised between $\sim-4 \%$ in the extensional volcanism of Pantelleria (Parello et al., 2000) and -3 to $0 \%$ o in the subduction-related volcanism of Vulcano Island (Capasso et al., 1997) and Etna (Allard et al., 1997; 
$\mathbf{a}$

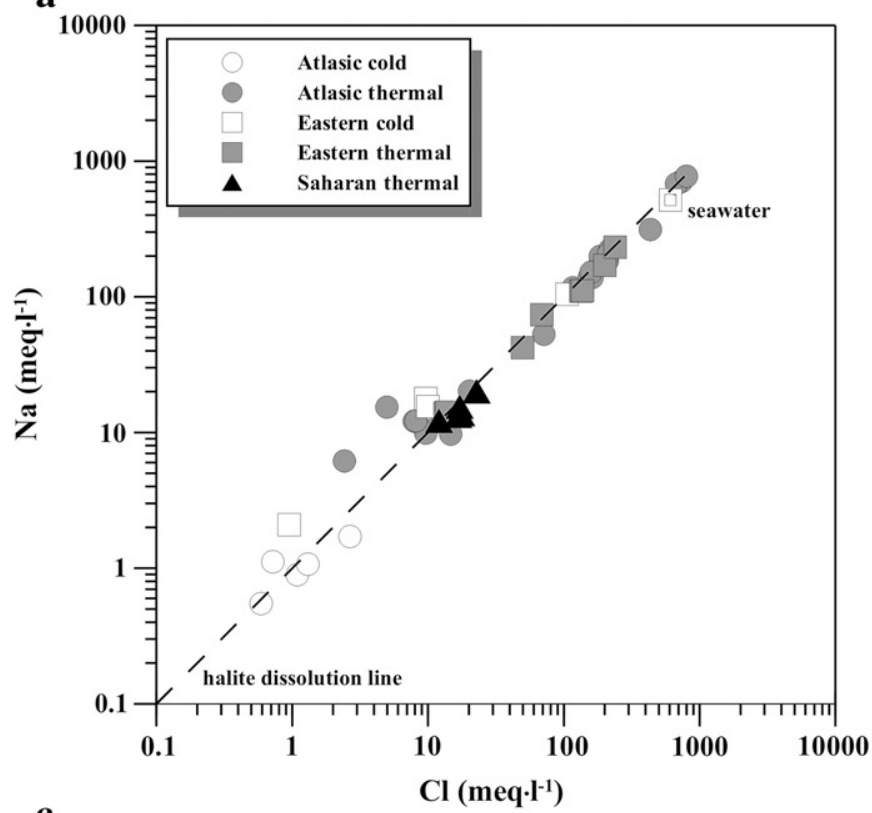

c

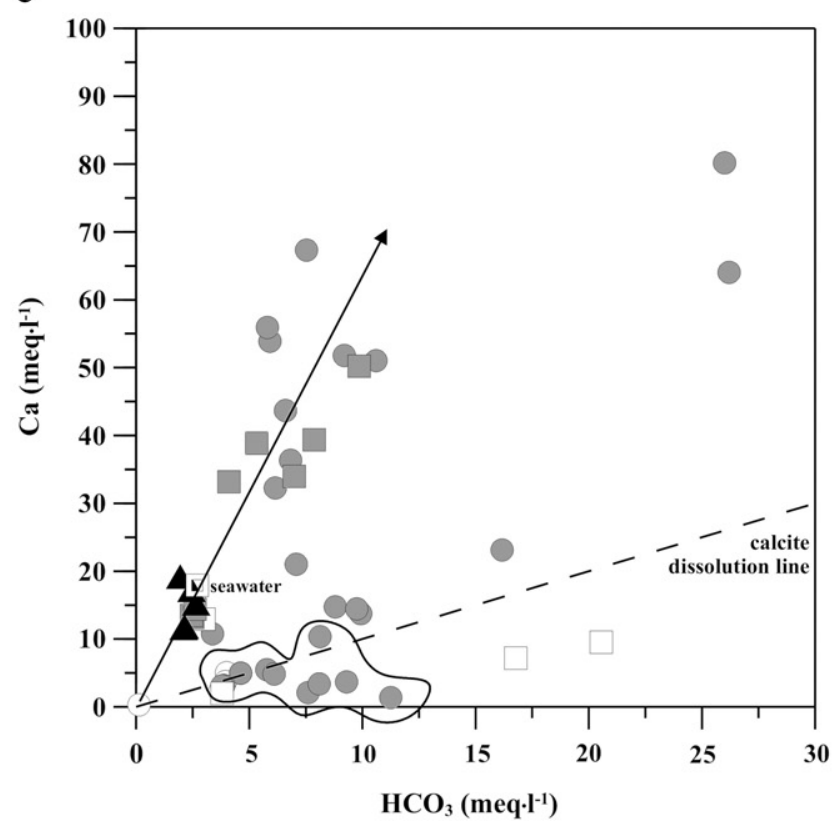

b

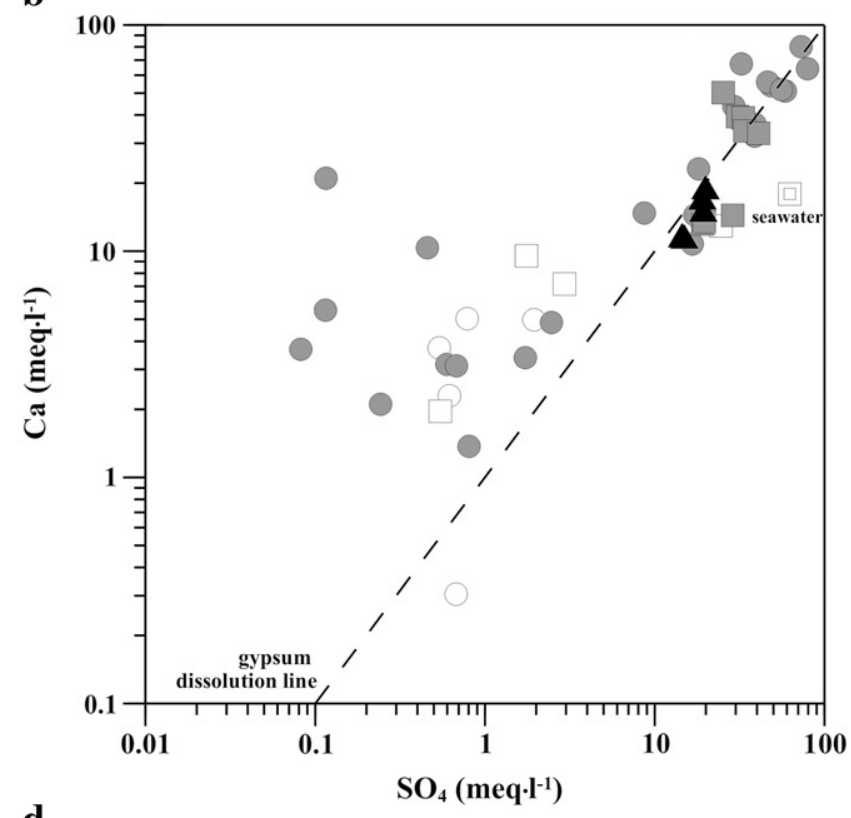

d

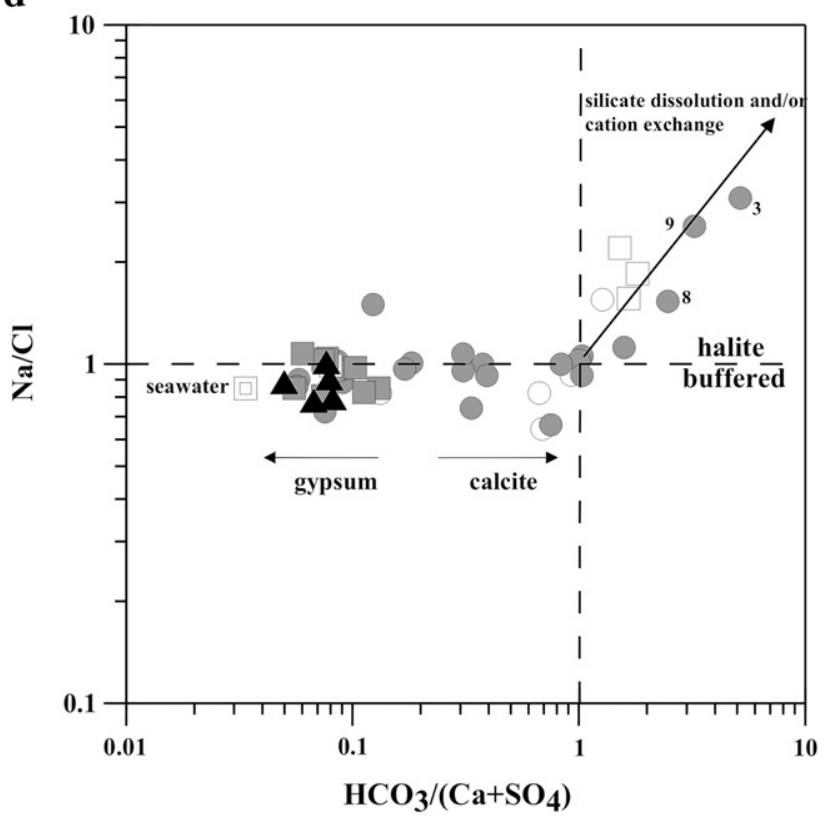

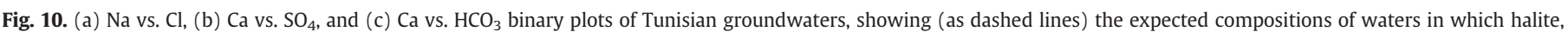

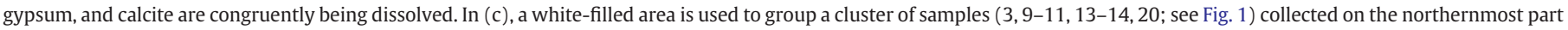

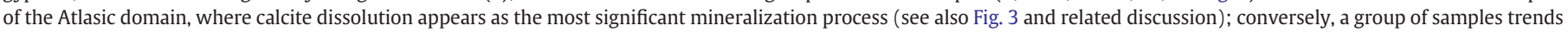

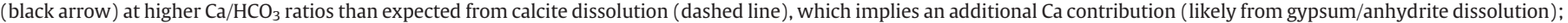
(d) $\mathrm{Na} / \mathrm{Cl}$ vs. $\mathrm{HCO}_{3} /\left(\mathrm{Ca}+\mathrm{SO}_{4}\right)$ diagram, summarizing the key source processes controlling the chemistry of Tunisian groundwaters. See text for explanation.

Chiodini et al., 2011). In addition, we note that our estimated range for abiogenic $\mathrm{CO}_{2}$ in Tunisia $\left(\delta^{13} \mathrm{C}_{\mathrm{CO} 2}\right.$ values between 0 and $+4 \%$ ) is more positive than the estimated deep- $\mathrm{CO}_{2}$ supply to groundwater systems of central ( $\delta^{13} \mathrm{C}_{\mathrm{CO} 2} \sim-3 \%$; Chiodini et al., 2000) to northcentral Italy $\left(\delta^{13} \mathrm{C}_{\mathrm{CO} 2}\right.$ from -3 to $+1 \%$; Frondini et al., 2008), where a crustally contaminated mantle has been proposed as the main source of abiogenic $\mathrm{CO}_{2}$ (Chiodini et al., 2004). Therefore, we argue that metamorphic decarbonation reactions in the deep crust are the most likely source of abiogenic carbon to the Tunisian groundwater system. In fact, assuming a range of $\delta^{13} \mathrm{C}$ for Tunisian carbonate rocks of -2 to $+2 \%$ o (Charisi and Schmitz, 1995), with an isotope enrichment factor $(\varepsilon)$ in the $\mathrm{CO}_{2}$-calcite system of 0.19 to $2.6 \%$ o in the $200-600{ }^{\circ} \mathrm{C}$ temperature range (Bottinga, 1969; Ohmoto and Rye, 1979), we calculate a $\delta^{13} \mathrm{C}$ for the $\mathrm{CO}_{2}$ produced during metamorphic decarbon- ation (Evans et al., 2008) ranging from -1.81 to $4.6 \%$, in total agreement with our calculated $\delta{ }^{13} \mathrm{C}_{\mathrm{CO} 2}$ range for Tunisian groundwaters.

The distribution maps of Fig. 8 highlight that this $\mathrm{CO}_{2}$-rich (Fig. 8a) and ${ }^{13} \mathrm{C}$-rich (Fig. 8b) volatile component is well represented in the entire northern sector of Tunisia, and more specifically in the northernmost parts of both the Atlasic (Tellian sector) and Eastern domains. This suggests that the intense tectonic activity related to Apennine-Maghrebide orogenesis, and the resulting high geothermal gradients, have likely created the favorable conditions for metamorphic decarbonation reactions to occur at regional scale. While thus the deep crust is likely sourcing the majority of carbon in the area, a striking link between shallow tectonic setting and degassing also clearly emerges, since most $\mathrm{CO}_{2}$-rich thermal manifestations are discharged in areas where a denser network of active faults (with 


\section{$\delta^{13} \mathbf{C}\left(\mathrm{CO}_{2}\right) \quad \mathbf{R} / \mathbf{R a}$}

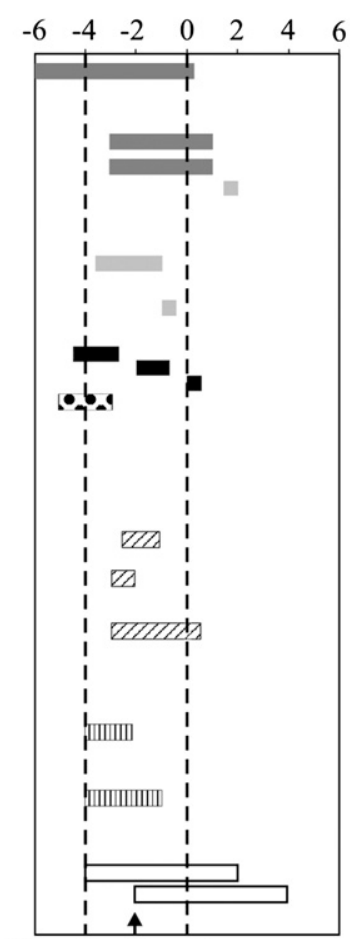

Site Ain Garci (sample 25)

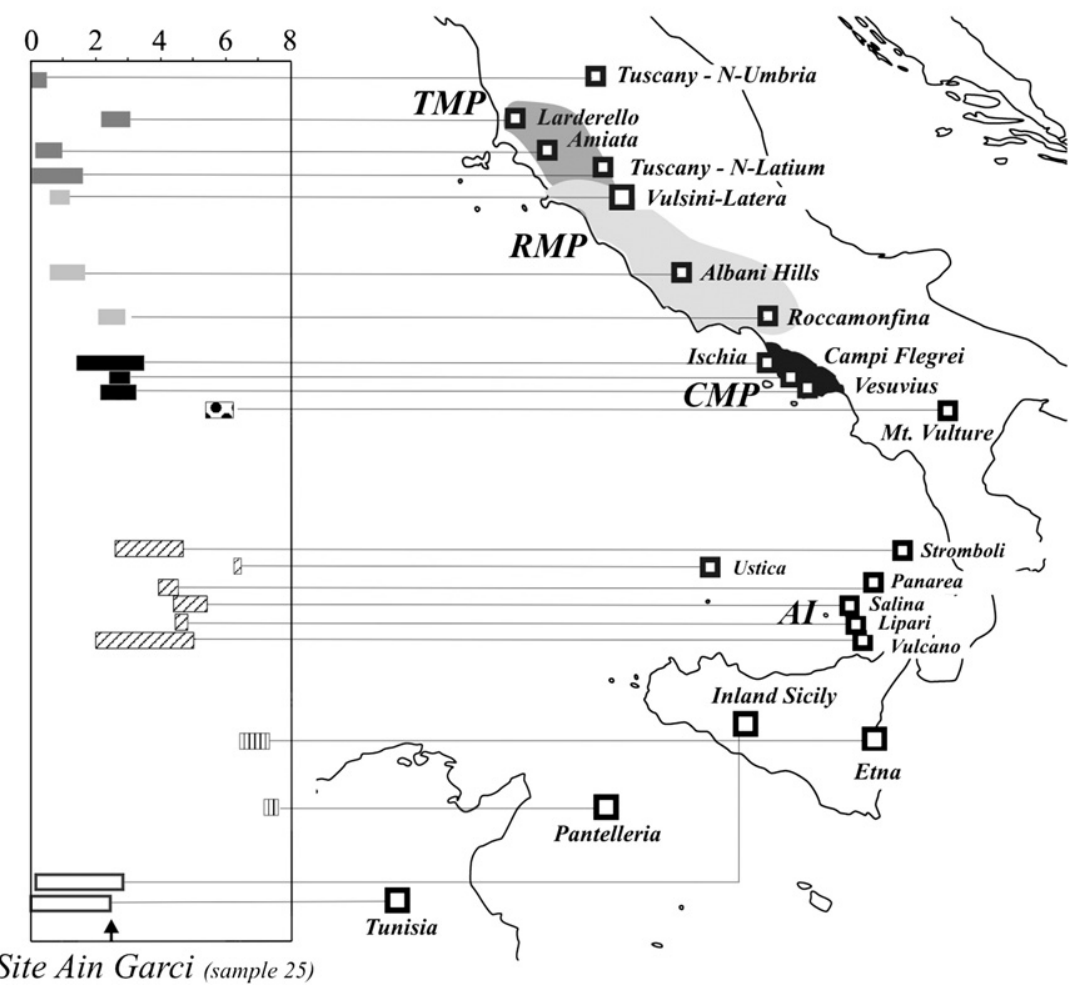

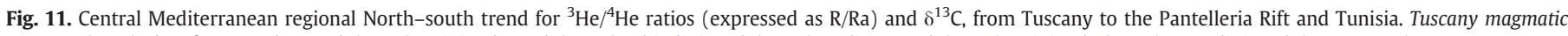

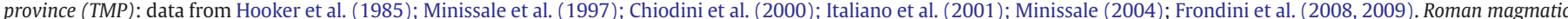

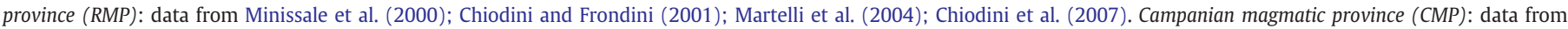

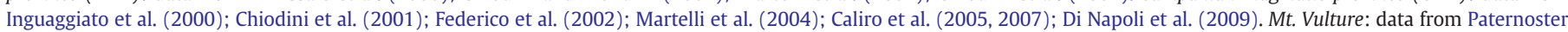

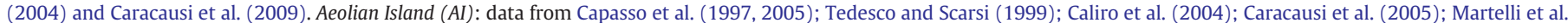

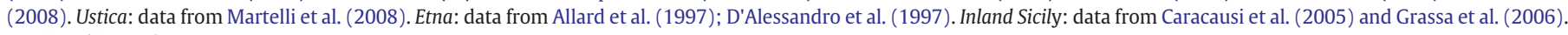
Tunisia: this work.

clear surface expression) favors the ascent of the deeply produced gasses.

In spite of the apparent prevailing crustal derivation of carbon, we acknowledge however that some extent of mantle $\mathrm{CO}_{2}$ contribution may also exist locally. In particular, sample 25 (Aïn Garci) has unusually $\delta^{13} \mathrm{C}_{\mathrm{CO} 2}$-poor composition $\left(\delta^{13} \mathrm{C}_{\mathrm{CO} 2}=-5.7 \%\right.$ o), at least relatively to similarly $\mathrm{CO}_{2}$-rich samples (e.g., sample $4, \delta{ }^{13} \mathrm{C}_{\mathrm{CO} 2}=$ $-4.3 \%$ ). This may be consistent with a contribution from some isotopically lighter abiogenic $\mathrm{CO}_{2}$. Since sample 25 has also an unusually high He isotope composition $(\mathrm{R} / \mathrm{Ra}=2.4)$, we conclude that some mantle-derived $\mathrm{CO}_{2}$ input has to be considered for the Eastern Tunisian domain.

\subsubsection{Helium isotopes distribution: links with geodynamics}

Helium results reveal some interesting spatial trends in the helium isotope composition (Fig.8c and d). Not unexpectedly, the lowest $\left({ }^{3} \mathrm{He} /{ }^{4} \mathrm{He}\right)_{x s}$ values $(0.01-0.02 \mathrm{Ra})$ are observed along an east-west Gabes-Tozeur line, associated with the old groundwaters of the $\mathrm{CI}$ aquifer. These ${ }^{3} \mathrm{He} /{ }^{4} \mathrm{He}$ values are comparable to the crustal production ratio (Ballentine and Burnard, 2002), with no detectable amount of mantle-derived ${ }^{3} \mathrm{He}$ added to this huge continental groundwater mass. This is in agreement with previous studies of helium isotopes in sedimentary basin, which conclude that tectonically-stable regions are essentially impermeable to mantle volatiles (Oxburgh et al., 1986; Marty et al., 2003; Kulongoski et al., 2008). In contrast with the ${ }^{3} \mathrm{He} /{ }^{4} \mathrm{He}$ ratios which are quite homogeneous, helium concentrations are more heterogeneous (Fig. 8c), ranging from $19.9 \times 10^{-8} \mathrm{~cm}^{3} \mathrm{STP} / \mathrm{g}$ in the Ras-el-Ain borehole (sample 38 ) to $404.8 \times 10^{-8} \mathrm{~cm}^{3} \mathrm{STP} / \mathrm{g}$ in El-Hamma (sample 39). This heterogeneity may be due to subsurface channeling of the crustal helium flux along discontinuities such as the El Hamma fault (Fig. 1). Further east, approaching the Gulf of Gabès and the island of Jerba, the $\left({ }^{3} \mathrm{He} /{ }^{4} \mathrm{He}\right)_{\mathrm{xs}}$ values rise up to $0.11 \mathrm{Ra}$. Although the region is characterized by large scale mixing between the CI groundwaters and the upper Djeffara local aquifer (Trabelsi et al., 2009), this cannot explain the higher aircorrected ${ }^{3} \mathrm{He} /{ }^{4} \mathrm{He}$ values, which may indeed be linked to the regional mantle- ${ }^{3} \mathrm{He}$ anomaly observed along the oriental margin of Tunisia, associated with the Sahel-Pelagian Block (see discussion below).

The low ${ }^{3} \mathrm{He} /{ }^{4} \mathrm{He}$ domain is not restricted to the Saharan Platform but actually extends to the entire Atlasic domain of central Tunisia (Fig. 8d). This Atlasic domain displays the highest helium concentrations : along the Gafsa Fault, helium concentrations of 1777 and $4723 \times 10^{-8} \mathrm{~cm}^{3} \mathrm{STP} / \mathrm{g}$ (the highest value of our data set) are observed in the two production wells (Z3 and Z1 respectively) of Sidi Ahmed Zarrouk (samples 34-35). High helium concentrations are also recorded southwest of Kairouan, in the two nearby sites of Ain Maamar (sample 29) $\left(2760 \times 10^{-8} \mathrm{~cm}^{3} \mathrm{STP} / \mathrm{g}\right)$ and Ain Chenama (sample 30) $\left(4060 \times 10^{-8} \mathrm{~cm}^{3} \mathrm{STP} / \mathrm{g}\right)$ both characterized by vigorous surface bubbling and high $\mathrm{CO}_{2}$ and methane contents. This high helium flux is associated with the tectonic knot at the intersection of the N-S Axis and the Mhrila-Cherichira fault zone - MCF (Fig. 1; Martinez et al., 1990; El Ghali et al., 2003). This again emphasizes the role of deep tectonic controls in channeling up and transporting deep crustal volatiles to shallow levels.

Northwards, low ${ }^{3} \mathrm{He} /{ }^{4} \mathrm{He}$ values between 0.02 and 0.05 Ra extend to the latitude of El Kef (sample 1). Further north, hot springs are more numerous and their ${ }^{3} \mathrm{He} /{ }^{4} \mathrm{He}$ ratios tend to increase significantly (although in some places ${ }^{3} \mathrm{He} /{ }^{4} \mathrm{He}$ values remain close to the crustal 
ratio) : $\left({ }^{3} \mathrm{He} /{ }^{4} \mathrm{He}\right)_{\mathrm{xs}}=0.13 \pm 0.08 \mathrm{Ra}$ for the cluster of hot springs located in the northwestern part of Tunisia, and up to $0.4 \mathrm{Ra}$ for the Ichkeul group (samples 15, 16 and 17) located at the extreme north of the country. This increase in the number of geothermal manifestations, accompanied by the presence of a weak but significant (5\%) mantle helium component (see Fig. 7), should be put in relation with the relatively high level of seismic activity across the whole area (Gueddiche et al., 1998), which could favor water circulation and mantle-helium leakage along active faults.

The eastern part of Tunisia displays higher $\left({ }^{3} \mathrm{He} /{ }^{4} \mathrm{He}\right)_{\mathrm{xs}}$ values (Fig. 8d) indicative of a substantial mantle volatile input. The highest (and sole value above the atmospheric ratio $-2.4 \mathrm{Ra}$ ) is recorded in the carbogaseous mineral water of Ain Garci (sample 25), in possible relation with the nearby volcanic outcrops of Djebel Fadeloun and Djebel Abid. Other $\left({ }^{3} \mathrm{He} /{ }^{4} \mathrm{He}\right)_{\mathrm{xs}}$ values range from $0.3 \mathrm{Ra}$ to $0.65 \mathrm{Ra}$. The uppermost values ( $0.6 \mathrm{Ra}$ and $0.65 \mathrm{Ra}$ ) correspond to the two sites nearest to Ain Garci (sample 25), suggesting the presence of a regional mantle ${ }^{3} \mathrm{He}$ anomaly. Helium concentrations range from 20 to $3800 \times 10^{-8} \mathrm{~cm}^{3} \mathrm{STP} / \mathrm{g}$, the latter being recorded in the abandoned well of Sidi Abdelhamid (sample 26) located on a major tectonic accident, the Kairouan-Sousse Fault (KSF) (Khomsi et al., 2004). Eastern Tunisia has been affected by an important tectonic activity since the Mesozoic times, with multiple magmatic intrusions (Mattoussi Kort et al., 2005). This northeastern part of the African plate, commonly referred to as the Pelagian Block (Burollet, 1978), extends from Tunisia to Sicily, and is thought as the southernmost foreland of the Apennine-Maghrebide orogenic system (Tavarnelli et al., 2004). This area is characterized by strong extensional tectonics (Pantelleria rift zone), crustal thinning, and present-day magmatic activity with $\left({ }^{3} \mathrm{He} /{ }^{4} \mathrm{He}\right)$ ratios around $8 \mathrm{Ra}$, typical of the upper mantle (Parello et al., 2000). This lithospheric stretching and decompression melting in the central portion of the Pelagian Block may facilitate the transport of mantle helium through the thinned crust away from the rift zone across the existing network of fractures and deep faults.

\section{Concluding remarks}

The chemical analysis of Tunisian groundwaters highlights the heterogeneity of the mineralization processes at work in Tunisia, as a consequence of the extremely variable geological and tectonic features. In terms of chemical composition, we conclude that dissolution of halite and gypsum plays a key control on groundwater chemistry. With respect to carbon and helium, this study represents the first isotopic characterization of Tunisian hydrothermal manifestations. Helium and carbon isotope systematics confirm the predominantly crustal origin for the gas phase supply to the Tunisian geothermal aquifers. Most samples are indeed characterized by radiogenic-type helium associated with a $\mathrm{CO}_{2}$ either predominantly organic (in the Saharan domain) or metamorphic in origin (in the Atlasic domain). As a whole, volatiles in Tunisian thermal fluids have He isotopic signatures resembling those characteristic of natural fluids from the northern Apennine area (Tuscany and N-Umbria), which are external to the Pleistocene Italian magmatic provinces, and which consistently have a prevalent crustal gas signature (Minissale et al., 2000). The prevalent crustal derivation of the fluids in most part of Tunisia is consistent with the absence of recent magmatism in the area (latest magmatic activity in Tunisia dates back to Miocene, related to the convergence between Africa and Eurasia). In eastern Tunisia, however, helium isotopes definitely support a more mantlelike derivation for $\mathrm{He}$ and $\mathrm{CO}_{2}$. In particular, the Ain Garci area is characterized by substantially higher helium isotopic compositions (up to $2.4 \mathrm{Ra}$, corresponding to $30 \%$ of mantle-derived helium), and by more negative isotopic composition of the dissolved carbon, consistent with a contribution from a lighter $\left(\delta^{13} \mathrm{C} \sim-2\right.$ to $\left.0 \%\right) \mathrm{CO}_{2}$ source. That mantle volatiles are to some extent involved in the eastern part of Tunisia would indeed be consistent with the fact that the Pelagian
Block (which eastern Tunisia is part of) has been deeply affected by extensional and transtensional tectonics since the opening of the Tethys Ocean (Tavarnelli et al., 2004), a process which is still ongoing in the Sicily Channel.

Our results add a new piece in the puzzle of the regional variability of deep fluid contributions in the western Mediterranean. Fig. 11 extends to Northern Africa the regional ( $\mathrm{N}$ to $\mathrm{S}$ ) isotopic trends, originally derived from the analysis of a variety of fluid types (natural gas discharges, dissolved gasses in groundwater, fluid inclusions in volcanic rocks) collected from seismically or volcanically active districts of northern-central (Tuscany) to southern (Sicily) Italy (Parello et al., 2000; Martelli et al., 2004, 2008). It shows that the Italian mantle gas anomaly only marginally extends to northwestern Africa, and that the north to south trend of increasing $\mathrm{R} / \mathrm{Ra}$ ratios, from the Tuscany, Roman and Campanian Magmatic Provinces $\left({ }^{3} \mathrm{He} /{ }^{4} \mathrm{He}\right.$ $<3 \mathrm{Ra})$ to Etna and Pantelleria $\left({ }^{3} \mathrm{He} /{ }^{4} \mathrm{He} \sim 7 \mathrm{Ra}\right)$, via the Aeolian Islands $\left({ }^{3} \mathrm{He} /{ }^{4} \mathrm{He} \sim 3-5 \mathrm{Ra}\right)$, apparently comes to an end at the latitude of the Pantelleria Rift. Our study finally strengthens the earlier conclusions of Mahood and Hildreth (1983) on the exceptional nature of Pantelleria volcanism, confirming that crustal thinning in the Sicily channel is unambiguously acting as a preferential path for upper mantle degassing at the regional scale.

\section{Acknowledgments}

This work benefited from the financial support of CNRS-DGRS (project no. 22636). We would like to thank the Tunisian Office of Thermalism for its cooperation and support. We also thank Sonia Falourd and Benedicte Minster for the water isotope measurements.

\section{References}

Aiuppa, A., Giudice, G., Gurrieri, S., Liuzzo, M., Burton, M., Caltabiano, T., McGonigle, A.J.S., Salerno, G., Shinohara, H., Valenza, M., 2008. Total volatile flux from Mount Etna. Geophys. Res. Lett. 35 (24), L24302 art. no.

Allard, P., Carbonelle, J., Dajlevic, D., Le Bronec, J., Morel, P., Robe, M.C., Maurenas, J.M., Faivre-Pierret, R., Martin, D., Sabroux, J.C., Zettwoog, P., 1991. Eruptive and diffuse emissions of $\mathrm{CO}_{2}$ from Mount Etna. Nature 351, 387-391.

Allard, P., D'Alessandro, W., Jean-Baptiste, P., Parello, F., Parisi, B., Flehoc, C., 1997. Mantle-derived helium and carbon in groundwaters and gas emanations of Mount Etna. Italy. Earth Planet. Sci. Lett. 148, 501-516.

Ballentine, C.J., Burnard, P.G., 2002. Production, release and transport of noble gases in the continental crust. In: Porcelli, D., Ballentine, C.J., Wieler, R. (Eds.), Noble gases in geochemistry and cosmochemistry: Rev. Mineral. Geochem., 47, pp. 481-538.

Ballentine, C.J., O'Nions, R.K., Oxburgh, E.R., Horvath, F., Deak, J., 1991. Rare-gas constraints on hydrocarbon accumulation, crustal degassing and groundwaterflow in the Pannonian Basin. Earth Planet. Sci. Lett. 105, 229-246.

Ballentine, CJ., Burgess, R. Marty, B., 2002. Tracing fluid origin, transport and interaction in the crust. In: Porcelli, D., Ballentine, C.J., Wieler, R. (Eds.), Noble gases in geochemistry and cosmochemistry: Rev. Mineral. Geochem., 47, pp. 539-614.

Bédir, M., 1995. Mécanismes géodynamiques des bassins associés aux couloirs de coulissement de la marge atlasique de la Tunisie. Thèse Doc. Etat, Tunis II, Tunisia, $416 \mathrm{pp}$.

Bédir, M., 1999. Subsurface structuring of the Eastern Mediterranean Tunisian basins: CIESM Workshop Series, 6, pp. 81-85.

Ben Ferjani, A., Burollet, P.F., Mejri, F., 1990. Petroleum Geology of Tunisia. Mem. E. T. A. P., vol. 1. Tunis, Tunisia. $194 \mathrm{pp}$.

Bishop, W.F., 1975. Geology of Tunisia and adjacent parts of Algeria and Lybia. Am. Assoc. Pet. Geol. Bull. 59, 413-450.

Bottinga, Y., 1969. Calculated fractionation factors for carbon and hydrogen isotope exchange in system calcite-carbon dioxide-graphite-methane-hydrogen-water vapor. Geochim. Cosmochim. Acta 33, 49-64.

Bouaziz, S., Barrier, E., Soussi, M., Turki, M., Zouari, H., 2002. Tectonic evolution of the northern african margin in Tunisia from paleostress data and sedimentary record. Tectonophysics 357, 227-253.

Bourg, C., Stievenard, M., Jouzel, J., 2001. Hydrogen and oxygen isotopic composition of aqueous salt solutions by gas-water equilibration method. Chem. Geol. 173, 331-337.

Bouri, S., Makni, J., Ben, Dhia H., 2008. A synthetic approach integrating surface and subsurface data for prospecting deep aquifers : the Southeast Tunisia. Environ. Geol. $54,1473-1484$

Brook, G.A., Folkoff, M.E., Box, E.O., 1983. A global model of soil carbon dioxide. Earth Surf. Processes Landforms 8, 79-88.

Burollet, P.F., 1956. Contribution à l'étude stratigraphique de la Tunisie centrale. Ann. Mines Geol., 18. Tunis, Tunisia. 345 pp. 
Burollet, P.F., 1978. Quaternary and recent movements in Kerkennah Island (Tunisie orientale). C.R. Acad. Sci., 286. Série D, Paris, pp. 1133-1136.

Caliro, S., Caracausi, A., Chiodini, G., Ditta, M., Italiano, F., Longo, M., Minopoli, C., Nuccio, P.M., Paonita, A., Rizzo, A., 2004. Evidence of a new magmatic input to the quiescent volcanic edifice of Panarea, Aeolian Islands. Italy. Geophys. Res. Lett. 31, 1-5.

Caliro, S., Chiodini, G., Avino, R., Cardellini, C., Frondini, F., 2005. Volcanic degassing at Somma-Vesuvio (Italy) inferred by chemical and isotopic signatures of groundwater. Appl. Geochem. 20 (6), 1060-1076.

Caliro, S., Chiodini, G., Moretti, R., Avino, R., Granieri, D., Russo, M., Fiebig, J., 2007. The origin of the fumaroles of La Solfatara (Campi Flegrei, South Italy). Geochim. Cosmochim. Acta 71, 3040-3055.

Capasso, G., Inguaggiato, S., 1998. A simple method for the determination of dissolved gases in natural waters. An application to thermal waters from Vulcano Island. Appl. Geochem. 13, 631-642.

Capasso, G., Favara, R., Inguaggiato, S., 1997. Chemical features and isotopic composition of gaseous manifestations on Vulcano Island, Aeolian Islands, Italy: an interpretative model of fluid circulation. Geochim. Cosmochim. Acta 61, 3425-3440.

Capasso, G., Favara, R., Inguaggiato, S., 2000. Interaction between fumarolic gases and thermal groundwaters at Vulcano Island (Italy): evidences from chemical composition of dissolved gases in waters. J. Volcanol. Geotherm. Res. 102, 309-318.

Capasso, G., Carapezza, M.L., Federico, C., Inguaggiato, S., Rizzo, A., 2005. Geochemical monitoring of the 2002-2003 eruption at Stromboli volcano (Italy): precursory changes in the carbon and helium isotopic composition of fumarole gases and thermal waters. Bull. Volcanol. 68, 118-134.

Caracausi, A., Favara, R., Italiano, F., Nuccio, P.M., Paonita, A., Rizzo, A., 2005. Active geodynamics of the central Mediterranean Sea: tensional tectonic evidences in western Sicily from mantle-derived helium. Geophys. Res. Lett. 32, L04312. doi:10.1029/2004GL021608.

Caracausi, A., Nuccio, P.M., Favara, R., Nicolosi, M., Paternoster, M., 2009. Gas hazard assessment at the Monticchio crater lakes of Mt. Vulture, a volcano in Southern Italy. Terra Nova 21, 83-87.

Castany, G., 1982. Bassin sédimentaire du Sahara septentrional (Algérie-Tunisie)Aquiféres du continental intercalare et du complexe terminal. Bull. Bur. Rec. Géol. Min. (BRGM) Sér. 2 (3), 127-147.

Celle-Jeanton, H., Zouari, K., Travi, Y., Daoud, A., 2001. Caractérisation isotopique des pluies en Tunisie. Essai de typologie dans la région de Sfax. C.R. Acad. Sci., 333. Séries IIA, Paris, pp. 625-631.

Charisi, S.D., Schmitz, B., 1995. Stable $\left(\delta^{13} \mathrm{C}, \delta^{18} \mathrm{O}\right)$ and strontium $\left({ }^{87} \mathrm{Sr} /{ }^{86} \mathrm{Sr}\right)$ isotopes through the Paleocene at Gebel Aweina, eastern Tethyan region. Palaeogeogr. Palaeoclimatol. Palaeoecol. 116, 103-129.

Chiodini, G., Frondini, F., 2001. Carbon dioxide degassing from the Albani Hills volcanic region, Central Italy. Chem. Geol. 177, 67-83.

Chiodini, G., Frondini, F., Cardellini, C., Parello, F., Peruzzi, L., 2000. Rate of diffuse carbon dioxide degassing estimated from carbon balance of regional aquifers: the case of central Apennine. Italy. J. Geophys. Res. 105, 8423-8434.

Chiodini, G., Marini, L., Russo, M., 2001. Geochemical evidences of high temperature hydrothermal brines at Vesuvio volcano (Italy). Geochim. Cosmochim. Acta 65, 2129-2147.

Chiodini, G., Cardellini, C., Amato, A., Boschi, E., Caliro, S., Frondini, F., Ventura, G., 2004. Carbon dioxide Earth degassing and seismogenesis in central and southern Italy. Geophys. Res. Lett. 31, L07615. doi:10.1029/2004GL019480.

Chiodini, G., Baldini, A., Barberi, F., Carapezza, M.L., Cardellini, C., Frondini, F., Granieri, D., Ranaldi, M., 2007. Carbon dioxide degassing at Latera caldera (Italy): evidence of geothermal reservoir and evaluation of its potential energy. J. Geophys. Res. 112, B12204, doi:10.1029/2006JB004896.

Chiodini, G., Caliro, S., Cardellini, C., Avino, R., Granieri, D., Schmidt, A., 2008. Carbon isotopic composition of soil $\mathrm{CO}_{2}$ efflux, a powerful method to discriminate different sources feeding soil $\mathrm{CO}_{2}$ degassing in volcanic-hydrothermal areas. Earth Planet. Sci. Lett. 274, 372-379.

Chiodini, G., Caliro, S., Aiuppa, A., Avino, R., Granieri, D., Moretti, R., Parello, F., 2011. First ${ }^{13} \mathrm{C} /{ }^{12} \mathrm{C}$ isotopic characterisation of volcanic plume $\mathrm{CO}_{2}$. Bull. Volc. 73 531-542. doi:10.1007/s00445-010-0423-2, in press.

Cornet, A., 1964. Introduction à l'hydrogéologie saharienne. Rev. Geog. Phys. Geol. Dyn. 6, 5-72.

Craig, H., 1961. Standards for reporting concentrations of deuterium and oxygen-18 in natural waters. Science 133, 1833-1834.

D'Alessandro, W., De Gregorio, S., Dongarrà, G., Gurrieri, S., Parello, F., Parisi, B., 1997. Chemical and isotopic characterization of the gases of Mount Etna (Italy). J. Volcanol. Geotherm. Res. 78, 65-76.

De Leeuw, G.A.M., Hilton, D.R., Fisher, T.P., Walker, J.A., 2007. The $\mathrm{He}-\mathrm{CO}_{2}$ isotope and relative abundance characteristics of geothermal fluids in El Salvador and Honduras: new constraints on volatile mass balance of the Central American Volcanic Arc. Earth Planet. Sci. Lett. 258, 132-146.

Deines, P., Langmuir, D., Harmon, R.S., 1974. Stable carbon isotope ratios and the existence of a gas phase in the evolution of carbonate groundwater. Geochim. Cosmochim. Acta 38, 1147-1164.

Di Napoli, R., Aiuppa, A., Bellomo, S., Brusca, L., D'Alessandro, W., Candela, E., Longo, M., Pecoraino, G., Valenza, M., 2009. A model for Ischia hydrothermal system: evidences from the chemistry of thermal groundwaters. J. Volcanol. Geotherm. Res. 186, 133-159.

Edmunds, W.M., Guendouz, A.H., Mamou, A., Moula, A., Shand, P., Zouari, K., 2003. Groundwater evolution in the Continental Intercalaire aquifer of southern Algeria and Tunisia: trace element and isotopic indicators. Appl. Geochem. 18, 805-822.
El Ghali, A., Bobier, C., Ben Ayed, N., 2003. Rôle du système de faille E-W dans l'évolution géodynamique de l'avant-pays de la chaîne alpine de Tunisie. Exemple de l'accident de Sbiba-Chérichira en Tunisie centrale. Bull. Soc. Geol. Fr. 174 (4), 373-381.

Evans, M.J., Derry, L.A., France-Lanord, C., 2008. Degassing of metamorphic carbon dioxide from the Nepal Himalaya. Geochem. Geophys. Geosyst. 9, Q04021. doi:10.1029/2007GC001796.

Favara, R., Grassa, F., Inguaggiato, S., Pecoraino, G., Capasso, G., 2002. A simple method to determine the $\delta^{13} \mathrm{C}$ of total dissolved inorganic carbon. Geofis. Int. 41, 313-320.

Federico, C., Aiuppa, A., Allard, P., Bellomo, S., Jean-Baptiste, P., Parello, F., Valenzia, M. 2002. Magma-derived gas influx and water-rock interactions in the volcanic aquifer of Mt Vesuvius. Italy. Geochim. Cosmochim. Acta 66, 963-981.

Fehdi, Ch., Rouabhia, Aek, Baali, F., Boudoukha, A., 2009. The hydrogeochemical characterization of Morsott-El Aouinet aquifer, Northeastern Algeria. Environ. Geol. 58, 1611-1620.

Frondini, F., Caliro, S., Cardellini, C., Chiodini, G., Morgantini, N., Parello, F., 2008. Carbon dioxide degassing from Tuscany and Northern Latium (Italy). Glob. Planet. Chang. 61, 89-102.

Frondini, F., Caliro, S., Cardellini, C., Chiodini, G., Morgantini, N., 2009. Carbon dioxide degassing and thermal energy release in the Monte Amiata volcanic-geothermal area (Italy). Appl. Geochem. 24, 860-875.

Gabtni, H., Jallouli, C., Mickus, K., Zouari, H., Turki, M.M., 2005. Geophysical Constraints on the location and nature of the North Saharan Flexure in Southern Tunisia. Pure Appl. Geophys. 162, 2051-2069.

Garcia, M.G., Del Hidalgo, M., Blesa, M.A., 2001. Geochemistry of groundwater in the alluvial plain of Tucuman province Argentina. J. Hydrol. 9, 597-610.

Gat, S.R., Carmi, I., 1970. Evolution of the isotopic composition of atmospheric waters in the Mediterranean sea area. J. Geophys. Res. 75, 3039-3048.

Giggenbach, W.F., 1988. Geothermal solute equilibria. Derivation of $\mathrm{Na}-\mathrm{K}-\mathrm{Mg}-\mathrm{Ca}$ geoindicators. Geochim. Cosmochim. Acta 52, 2749-2765.

Giggenbach, W.F., Sano, Y., Wakita, H., 1993. Isotopic composition of helium, and $\mathrm{CO}_{2}$ and $\mathrm{CH}_{4}$ contents in gases produced along the New Zealand part of a convergent plate boundary. Geochim. Cosmochim. Acta 57, 3427-3455.

Goff, F., Janik, C.J., 2000. Geothermal systems. In: Sigurdsson, H., Houghton, B., McNutt S., Rymer, H., Stix, J. (Eds.), Encyclopedia of Volcanoes. Academic Press, San Diego, CA, pp. 817-834.

Gonfiantini, R., Conrad, G., Fontes, JCh., Sauzay, G., Payne, B.R., 1974. Etude isotopique de la nappe du Continental Intercalaire et de ses relations avec les autres nappes du Sahara septentrional (Isotopic investigation of the Continental Intercalaire aquifer and its relationship with other aquifers in the northern Sahara). Isotope Techniques in Groundwater Hydrology, vol. 1. IAEA SM-182/25, Vienna, pp. 227-241.

Graham, D.W., 2002. Noble gas isotope geochemistry of Mid-Ocean Ridge and Ocean Island Basalts: characterization of mantle source reservoirs. In: Porcelli, D. Ballentine, C.J., Wieler, R. (Eds.), Noble gases in geochemistry and cosmochemistry: Rev. Mineral. Geochem., 47, pp. 247-317.

Grassa, F., Capasso, G., Favara, R., Ingaggiato, S., 2006. Chemical and isotopic composition of waters and dissolved gases in some thermal springs of Sicily and adjacent volcanic islands. Italy. Pure Appl. Geophys. 163, 781-807.

Griesshaber, E., O'Nions, R.K., Oxburgh, E.R., 1992. Helium and carbon isotope systematics in crustal fluids from the Eiffel, the Rhine Graben and Black Forest Chem. Geol. 99, 213-235.

Gueddiche, M., Ben Ayed, N., Mohammadioun, G., Mohammadioun, B., Elghali, A. Chekhma, H., Diament, M., Dubois, J., 1998. Etude sismotectonique de la Tunisie nord-orientale. Bull. Soc. Geol. Fr. 169, 789-796.

Guendouz, A., Michelot, J.L., 2006. Chlorine-36 dating of deep groundwater from northern Sahara. J. Hydrol. 328, 572-580.

Guendouz, A., Moulla, A.S., Edmunds, W.M., Shand, P., Zouari, K., Mamou, A., 1997 Palaeoclimatic information contained in groundwaters of the Grand Erg Oriental North Africa. Int Symp Isotope Techniques in the Study of Past and Curren Environmental Changes in the Hydrosphere and Atmosphere, Vienna, 14-18 April 1997. IAEA-SM-349-/43.

Guendouz, A., Moulla, A.S., Edmunds, W.M., Zouari, K., Shand, P., Mamou, A., 2003. Hydrogeochemical and isotopic evolution of water in the Complex Terminal aquifer in the Algerian Sahara. Hydrogeol. J. 11, 483-495.

Güleç, N., Hilton, D.R., Mutlu, H., 2002. Helium isotope variations in Turkey: relation to tectonics, volcanism and recent seismic activities. Chem. Geol. 187, 129-142.

Hamada and Tanaka, 2001. Dynamics of carbon dioxide in soil profiles based on longterm field observation. Hydrol. Process. 15, 1829-1845.

Hilton, D.R., 1996. The helium and carbon isotope systematics of a continental geothermal system: results from monitoring studies at Long Valley caldera (California, USA). Chem. Geol. 127, 269-295.

Hilton, D.R., Fischer, T.P., Marty, B., 2002. Noble gases and volatile recycling at subduction zones. In: Porcelli, D., Ballentine, C.J., Wieler, R. (Eds.), Noble gases in geochemistry and cosmochemistry: Rev. Mineral. Geochem., 47, pp. 319-370.

Hooker, P.J., Bertrami, R., Lombardi, S., O'Nions, R.K., Oxburgh, E.R., 1985. Helium-3 anomalies and crust-mantle interactions in Italy. Geochim. Cosmochim. Acta 49 2505-2513.

Hulston, J.R., Hilton, D.R., Kaplan, I.R., 2001. Helium and carbon isotope systematics of natural gases from Taranaki Basin, New Zealand. Appl. Geochem. 16, 419-436.

Inguaggiato, S., Pecoraino, G., D'Amore, F., 2000. Chemical and isotopic characterization of fluid manifestations of Ischia Island. J. Volcanol. Geotherm. Res. 99, 151-178.

Inoubli, N., Gouasmia, M., Gasmi, M., Mhamdi, A., Ben Dhia, H., 2006. Integration of geological, hydrochemical and geophysical methods for prospecting thermal water resources: The case of the Hmeïma region (Central -Western Tunisia). J. Afr. Earth. Sci. $46,180-186$. 
Irwin, W.P., Barnes, I., 1980. Tectonic relations of carbon dioxide discharges and earthquakes. J. Geophys. Res. 85, 3115-3121.

Italiano, F., Martinelli, G., Nuccio, P.M., 2001. Anomalies of mantle-derived helium during the 1997-1998 seismic swarm of Umbria-Marche. Italy. Geophys. Res. Lett. 28, 839-842.

Iundt, F., 1971. Potentiel géothermique de la Tunisie. Etude géochimique. Bureau de Recherches Géologiques et Minières. Service Géologique National, Orléans, France.

Jean-Baptiste, P., Mantisi, F., Dapoigny, A., Stievenard, M., 1992. Design an performance of a mass spectrometric facility for measuring helium isotopes in natural waters and for low-level tritium determination by the ${ }^{3} \mathrm{He}$ ingrowth method. Appl. Radiat. Isot. 43, 881-891.

Jellouli, A., 2002. La Tunisie thermale. Office du thermalisme, Tunis. 193 pp.

Kamel, S., Dessi, L., Zouari, K., Abidi, B., 2005. Geochemical and isotopic investigation of the aquifer system in the Djerid-Nefzaoua basin, southern Tunisia. Environ. Geol. 49, 159-170.

Khomsi, S., Bédir, M., Zouari, H., Ben Jemia, G., 2004. Mise en évidence et analyse d'une structure atlasique ennoyée au front de la Chaîne alpine tunisienne. C.R. Geosci. 336, 1293-1300.

Khomsi, S., Bédir, M., Soussi, M., Ben Jemia, G., Ben Ismail-Lattrache, K., 2007. Réponse au commentaire de N. Brahim et E. Mercier à propos de l'article de S. Khomsi et al. C.R. Geoscience 338 (1-2) (2006) 41-49. C.R. Geoscience 339, 173-177.

Kipfer, R., Aeschbach-Hertig, W., Peeters, F., Stute, M., 2002. Noble gases in lakes and ground waters. In: Porcelli, D., Ballentine, C.J., Wieler, R. (Eds.), Noble gases in geochemistry and cosmochemistry: Rev. Mineral. Geochem., 47, pp. 615-700.

Kouzana, L., Ben Mammou, A., Sfar Felfoul, M., 2009. Seawater intrusion and associated processes: case of the Korba aquifer (Cap Bon, Tunisia). C.R. Geosci. 341, 21-35.

Kouzana, L., Benassi, R., Ben Mammou, A., Sfar Felfoul, M., 2010. Geophysical and hydrochemical study of the seawater intrusion in Mediterranean semi arid zones. Case of the Korba coastal aquifer (Cap-Bon, Tunisia). J. Afr. Earth. Sci. 58, $242-254$.

Kulongoski, J.T., Hilton, D.R., Cresswell, R.G., Hostetler, S., Jacobson, G., 2008. Helium-4 characteristics of groundwaters from Central Australia : comparative chronology with Chlorine-36 and Carbon-14 dating techniques. J. Hydrol. 348, 176-194.

Laaridhi-Ouazaa, N., 1994. Etude minéralogique et géochimique des épisodes magmatiques mésozoïques et miocénes de la Tunisie. Thèse Doc. Etat, Univ. Tunis II, Tunisia, $466 \mathrm{pp}$

Langelier, W.F., Ludwig, H.F., 1942. Graphical method for indicating the mineral character of natural waters. J. Am. Waterworks Assoc. 34, 335-352.

Lollar, B.S., Ballentine, C.J., 2009. Insights into deep carbon derived from noble gases. Nat. Geosci. 2, 543-547.

Lollar, B.S., Ballentine, C.J., ONions, R.K., 1997. The fate of mantle-derived carbon in a continental sedimentary basin: integration of $\mathrm{C} / \mathrm{He}$ relationships and stable isotope signatures. Geochim. Cosmochim. Acta 61, 2295-2307.

Mahood, G.A., Hildreth, W., 1983. Nested calderas and trapdoor uplift at Pantelleria, Strait of Sicily. Geology 11, 722-726.

Martelli, M., Nuccio, P.M., Stuart, F.M., Burgess, R., Ellam, R.M., Italiano, F., 2004 Helium-strontium isotope constraints on mantle evolution beneath the Roman Comagmatic Province. Italy. Earth Planet. Sci. Lett. 224, 295-308.

Martelli, M., Nuccio, P.M., Stuart, F.M., Di Liberto, V., Ellam, R.E., 2008. Constrains on mantle source and interactions from He-Sr isotope variation in Italian PlioQuaternary volcanism. Geochem. Geophys. Geosyst. 9, Q02001. doi:10.1029/ 2007GC001730.

Martinez, C., Andrieux, J., Truillet, R., Ben Jemiaa, M., 1990. Les structures synsédimentaires miocènes en compression associées au décrochement dextre MhrilaChérichira (Tunisie centrale). Bull. Soc. Geol. Fr. 6 (1), 167-176.

Marty, B., Jambon, A., 1987. C ${ }^{3} \mathrm{He}$ in volatile fluxes from the solid earth : implication for carbon geodynamics. Earth Planet. Sci. Lett. 83, 16-26.

Marty, B., Gunnlaugsson, E., Jambon, A., Oskarsson, N., Ozima, M., Pineau, F., Torssander P., 1991. Gas geochemistry of geothermal fluids, the Hengill area, southwest rift zone of Iceland. Chem. Geol. 91, 207-225.

Marty, B., O'Nions, R.K., Oxburgh, E.R., Martel, D., Lombardi, S., 1992. Helium isotopes in Alpine regions. Tectonophysics 206, 1-8.

Marty, B., Pik, R., Gezahegn, Y., 1996. Helium isotopic variations in Ethiopian plume lavas: nature of magmatic sources and limit on lower mantle contribution. Earth Planet. Sci. Lett. 144, 223-237.

Marty, B., Dewonck, S., France-Lanord, C., 2003. Geochemical evidence for efficient aquifer isolation over geological timeframes. Nature 425, 55-58.

Mata, J., Moreira, M., Doucelance, R., Ader, M., Silva, L.C., 2010. Noble gas and carbon isotopic signatures of Cape Verde oceanic carbonatites: implications for carbon provenance. Earth Planet. Sci. Lett. 291, 70-83.

Mattoussi Kort, H., Laridi Ouazaa, N., Kassaa, S., Beji Sassi, A., 2005. Thermal effects of magmatic activity on clay sediments in eastern Tunisia. Mineral Deposit Research: Meeting the Global Challenge 2005, Session 9 999-1001. doi:10.1007/3-54027946-6_255.

Mauduit, F., 1978. Le volcanisme néogène de la Tunisie continentale. Thèse 3ème cycle. Univ. Paris - Sud (Orsay).
Meddeb, M. N., 1993. Potentialités géothermiques de la Tunisie septentrionale. Thèse de $3^{\text {ème }}$ cycle, Laboratoire Hydrogéologie - Géothermie, Univ. Tunis II F.S.T. et E.N.I.S. Minissale, A., 2004. Origin, transport and discharge of $\mathrm{CO}_{2}$ in central Italy. Earth Sci. Rev. 66, 89-141.

Minissale, A., Evans, W.C., Magro, G., Vaselli, O., 1997. Multiple source components in gas manifestations from north-central Italy. Chem. Geol. 142, 175-192.

Minissale, A., Magro, G., Martinelli, G., Vaselli, O., Tassi, F., 2000. Fluid geochemical transect in the Northern Apennines (central-northern Italy): fluid genesis and migration and tectonic implications. Tectonophysics 319, 199-222.

Mlayah, A., Ferreira da Silva, E., Rocha, F., Ben Hamza, Ch., Charef, A., Noronha, F., 2009. The Oued Mellègue: Mining activity, stream sediments and dispersion of base metals in natural environments, North-western Tunisia. J. Geochem. Explor. 102, 27-36.

Mutlu, H., Güleç, N., Hilton, D.R., 2008. Helium-carbon relationships in geothermal fluids of western Anatolia. Turkey. Chem. Geol. 247, 305-321.

Ohmoto, H., Rye, R.O., 1979. Isotopes of sulfur and carbon. In: Barnes, H.L. (Ed.), Geochemistry of Hydrothermal Ore Deposits, pp. 509-567.

Oxburgh, E.R., O'Nions, R.K., Hill, R.I., 1986. Helium isotopes in sedimentary basins. Nature 324, 632-635.

Parello, F., Allard, P., D'Alessandro, W., Federico, C., Jean-Baptiste, P., Catani, O., 2000. Isotope geochemistry of Pantelleria volcanic fluids, Sicily Channel rift: a mantle volatile end-member for volcanism in southern Europe. Earth Planet. Sci. Lett. 180, 325-339.

Parkhurst, D.L., Appelo, C.A.J., 1999. User's guide to PHREEQC (Version 2) - a computer program for speciation, batch-reaction, one-dimensional transport, and inverse geochemical calculations. USGS: Water Resour. Invest. Rep., 99, p. 4259.310 pp.

Paternoster, M., 2004, Mt. Vulture volcano (Italy): a geochemical contribution to the origin of fluids and to a better definition of its geodynamic setting. PhD dissertation, Università di Palermo.

Perthuisot, V., 1978. Dynamique et pétrogenèse des extrusions triasiques en Tunisie septentrionale. PhD thesis, Ecole Normale Supérieure, Paris, 312 pp.

Pik, R., Marty, B., 2009. Helium isotopic signature of modern and fossil fluids associated with the Corinth rift fault zone (Greece): implication for fault connectivity in the lower crust. Chem. Geol. 266, 67-75.

Polyak, B.G., Tolstikhin, I.N., Kamensky, I.L., Yakovlev, L.E., Marty, B., Cheshko, A.L., 2000. Helium isotopes, tectonics and heat flow in the Northern Caucasus. Geochim. Cosmochim. Acta 64, 1925-1944.

Rouvier, H., 1977. Géologie de l'extrême Nord-tunisien: tectoniques et paléogéographies superposées à l'extrémité orientale de la chaîne Nord-Maghrébine. PhD Thesis, Univ. P.M. Curie, Paris, France, 1000 pp.

Sadki, O., 1998. Etude de systèmes hydrothermaux du Nord de la Tunisie. Géochimie des interactions eaux-roches et circulation hydrothermale. PhD thesis, Laboratoire de géochimie et géologie de l'environnement, Univ. Tunis II F.S.T. et E.N.I.S.

Sano, Y., Williams, S., 1996. Fluxes of mantle and subducted carbon along convergent plate boundaries. Geophys. Res. Lett. 23, 2749-2752.

Stievenard, M., Delmotte, M., Jouzel, J., et al., 1994. Mass spectrometric analysis of water stable isotopes - reconstruction of past climates from polar ice cores. Analusis 22, M21-M24.

Stuart, F.M., Lass-Evans, S., Fitton, J.G., Ellam, R.M., 2003. High ${ }^{3} \mathrm{He} /{ }^{4} \mathrm{He}$ ratios in picritic basalts from Baffin Island and role of a mixed reservoir in mantle plumes. Nature 424, 57-59.

Stumm, W., Morgan, J.J., 1996. Aquatic chemistry: chemical equilibria and rates in natural waters. Wiley-Interscience publication.

Tavarnelli, E., Butler, R.W.H., Decandia, F.A., Calamita, F., Grasso, M., Alvarez, W., Renda, P., 2004. Implications of fault reactivation and structural inheritance in the cenozoic tectonic evolution of Italy. IGC: Bollettino della Societa Geologica Italiana, Special Volume, 32, pp. 209-222. 0037-8763.

Tedesco, D., Scarsi, P., 1999. Intensive gas sampling of noble gases and carbon at Vulcano Island (southern Italy). J. Geophys. Res. 104, 10499-10510. doi:10.1029/ 1998JB 900066

Trabelsi, R., Zaïri, M., Smida, H., Ben Dhia, H., 2005. Salinization of coastal aquifers : case of the North Sfax Sahel aquifer, Tunisia. C.R. Geosci. 337, 515-524.

Trabelsi, R., Zaïri, M., Ben Dhia, H., 2007. Groundwater salinization of the Sfax superficial aquifer, Tunisia. Hydrogeol. J. 15, 1341-1355.

Trabelsi, R., Kacem, A., Zouari, K., Rozanski, K., 2009. Quantifying regional groundwater flow between Continental Intercalaire and Djeffara aquifers in southern Tunisia using isotope methods. J. Environ. Geol. 58, 171-183.

Wigley, T.M.L., Plummer, L.N., Pearson Jr., F.J., 1978. Mass transfer and carbon isotope evolution in natural waters systems. Geochim. Cosmochim Acta 42, 1117-1139.

Yermani, M., Zouari, K., Michelot, J.L., Mamou, A., Moumni, L., 2003. Geochemical approach to the functioning of the Gafsa North deep aquifer (central Tunisia). Hydrol. Sci. J. J. Sci. Hydrol. 48, 95-108.

Zhang, J., Quay, P.D., Wilbur, D.O., 1995. Carbon isotope fractionation during gas-water exchange and dissolution of CO2. Geochim. Cosmochim. Acta 59, 107-114

Zouari, K., Chkir, N., Ouda, B., 2003. Palaeoclimatic variation in Maknassi basin (central Tunisia) during Holocene period using pluridisplinary approaches. IAEA Tech. Report, pp. 80-88. Vienna, Austria. 